Article

\title{
A Multichannel FRA-Based Impedance Spectrometry Analyzer Based on a Low-Cost Multicore Microcontroller
}

\author{
Arturo Sanchez-Gonzalez, Nicolas Medrano *(D), Belen Calvo and Pedro A. Martinez \\ Group of Electronic Design, Aragon Institute for Engineering Research, University of Zaragoza, 50009 Zaragoza, \\ Spain; asgonzalez@unizar.es (A.S.-G.); becalvo@unizar.es (B.C.); pemar2@unizar.es (P.A.M.) \\ * Correspondence: nmedrano@unizar.es; Tel.: +34-876-553-358
}

Received: 9 November 2018; Accepted: 21 December 2018; Published: 1 January 2019

check for updates

\begin{abstract}
Impedance spectrometry (IS) is a characterization technique in which a voltage or current signal is applied to a sample under test to measure its electrical behavior over a determined frequency range, obtaining its complex characteristic impedance. Frequency Response Analyzer (FRA) is an IS technique based on Phase Sensitive Detection (PSD) to extract the real and imaginary response of the sample at each input signal, which presents advantages compared to FFT-based (Fast Fourier Transform) algorithms in terms of complexity and speed. Parallelization of this technique has proven pivotal in multi-sample characterization, reducing the instrumentation size and speeding up analysis processes in, e.g., biotechnological or chemical applications. This work presents a multichannel FRA-based IS system developed on a low-cost multicore microcontroller platform which both generates the required excitation signals and acquires and processes the output sensor data with a minimum number of external passive components, providing accurate impedance measurements. With a suitable configuration, the use of this multicore solution allows characterizing several impedance samples in parallel, reducing the measurement time. In addition, the proposed architecture is easily scalable.
\end{abstract}

Keywords: electrochemical impedance spectroscopy; FRA; multichannel acquisition; impedance spectrometry; microcontroller

\section{Introduction}

Electrochemical impedance spectroscopy (EIS), in which a sinusoidal signal is applied to a sample under test to evaluate in a determined frequency range its complex impedance-typically modeled as a Randles cell [1] —is a powerful sensing technique that has experienced significant development over the last years due to its broad range of applications. These span from the biotechnological field (rapid detection of foodborne pathogenic bacteria, detection and enumeration of E. coli bacteria in milk samples, real-time detection of milk adulteration, food control, antibiotic susceptibility testing of E. coli and characterization of cellular dielectric properties for cell health evaluation [2-7]), to the characterization of materials (microstructures, dielectric materials, corrosion evaluation, [8-11]), as well as electrical circuit testing and characterization of electrical systems, batteries, and photovoltaic cells $[8,12,13]$. Unlike other electrochemical techniques, such as potentiometric and amperometric sensing (based on DC voltage and current excitation, respectively [14]), the characterization of a sample by its impedance changes over frequency requires a small stimulus signal, reducing the risk of sample damaging, which is a key point in biological measurement and characterization applications. Information can be then recovered using different readout techniques, being the Fast Fourier Transform (FFT) and the Frequency Response Analyzer (FRA) the two most commonly used. The latter one is 
based on synchronous demodulation, that is, it relies on phase sensitive detection (PSD) or quadrature modulators to extract the real and imaginary response of the sensor at each input signal $f_{\text {in }}$ while noise signals at other frequencies are rejected (Figure 1), presenting advantages compared to FFT algorithms in terms of complexity and speed $[15,16]$. Thus, the FRA-EIS is a more suitable choice to accomplish an autonomous low-cost real-time multichannel impedance spectroscopy analyzer.

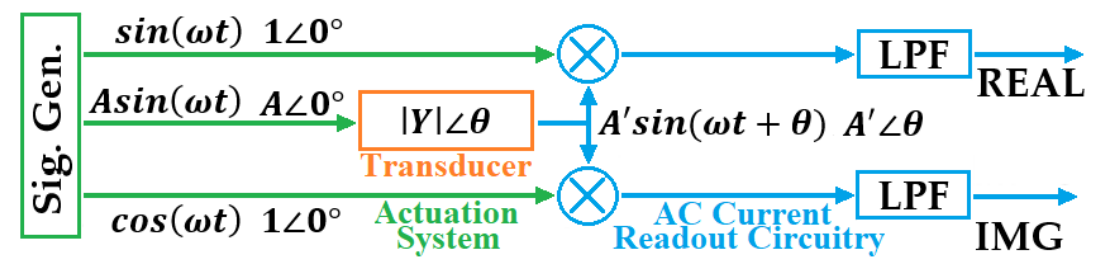

Figure 1. Single channel (Frequency Response Analyzer) FRA-based impedance spectroscopy (IS) system block diagram.

In this attempt, while electrochemical transducers take advantage of Complementary Metal-Oxide-Semiconductor (CMOS) processes to implement the required Micro-Electro-Mechanical Systems (MEMS [17]), the rest of components that conform the data acquisition chain (signal stimuli generators, conditioning, pre-processing and digitization electronics) are still bulky benchtop instruments, making EIS almost exclusively a measurement technique for biochemical, biological, or quality control laboratories, but hindering its use closer to the sampling sources, as milk farms, in food production chains or portable laboratories for on-site tests.

Recent publications in the scientific and technical literature are reporting EIS systems partially implemented using CMOS technologies by addressing specific low-power low-size design techniques to take advantage of the features that miniaturization can provide to the system in terms of portability and high parallelism in the measurement processes [18-20]. These cases succeed in the integration of competitive read-out channels, but the generated real/imaginary analog data must be finally digitized to be processed by a digital processing unit (a microcontroller, a digital signal processor or an external computer), while the generation of the required excitation and control signals are usually assigned either to external resources (commercial waveform generators that provide flexibility in exchange of large size and high power consumption, not being compatible with portability), or small size custom integrated oscillators [21], with exhibit frequency tuning and linearity limitations, especially at high frequencies. Hence, although being fundamental components, both the generation and digitization blocks are not usually considered in the power consumption estimation of the EIS system, thus giving partial information of the real energy required by a complete measurement unit.

In order to reduce electronics complexity, alternative EIS approaches are based on the direct transform of impedance to digital values using impedance-to-digital or dual-slope multiplying ADC (DS-MADC) techniques, achieving accuracies below 10 bits [22,23]. With the goal of further reduce electronics complexity, in order to attain a self-contained low-cost measurement system that renders a true portability while preserving high recovery performance, this paper proposes the complete digitalization of the EIS system through a microcontroller-based FRA implementation, applied to impedance spectroscopy for frequencies in the range of cellular characterization, from $1.1 \mathrm{mHz}$ to $10 \mathrm{kHz}$. The proposed system uses the internal resources of a Propeller processor core from Parallax [24], to both generate the excitation and control signals required in the process, and to map the read-out and recovery algorithms for the sensed analog signals, so that with minimum additional passive components, it can recover an impedance value with 12-bit accuracy. In addition, the use of this low-cost multicore processor allows for parallelization of the actuation and signal recovery, implementing a multi-channel compact IS instrument on a single microcontroller, able to perform up to 7 real-time in-situ parallel impedance measurements if driven by the same signal generator, or up 4 completely independent parallel impedance measurements; these values can be further extended by accordingly extending the number of cores to allow further parallelization. 
This paper is structured as follows: Section 2 describes the proposed FRA-based impedance analyzer, detailing the implementation and the experimental characterization of both the actuation and the signal acquisition blocks. Section 3 validates the recovery performance of the proposed IES system applied to an impedance modeling a bilayer lipid membrane. Finally, Section 4 discusses the proposed approach.

\section{Proposed EIS System}

The proposed EIS system relies on the use of a single Parallax Propeller microcontroller, characterized by working at up to $80 \mathrm{MHz}$ clock frequency. It presents 8 independent cores plus an additional hub, in charge of controlling the access of each core to the common microcontroller resources (32 kB Main RAM or $32 \mathrm{kB}$ Main ROM), applying a Round Robin schedule. Each core features a video generator, a local 2 kB RAM, and two Counter Modules with Phase-Locked Loops (PLLs) and 32 operation modes (Figure 2). From a software point of view, the microcontroller can be programmed in C, in its own high-level programming language SPIN, or in low-level Propeller Assembly Language (PASM). In order to achieve a suitable implementation of a FRA-based EIS system, optimizing the hardware resources and their access at the rate needed to generate and recover signals of a reasonable frequency, an integral programming in PASM has been adopted.

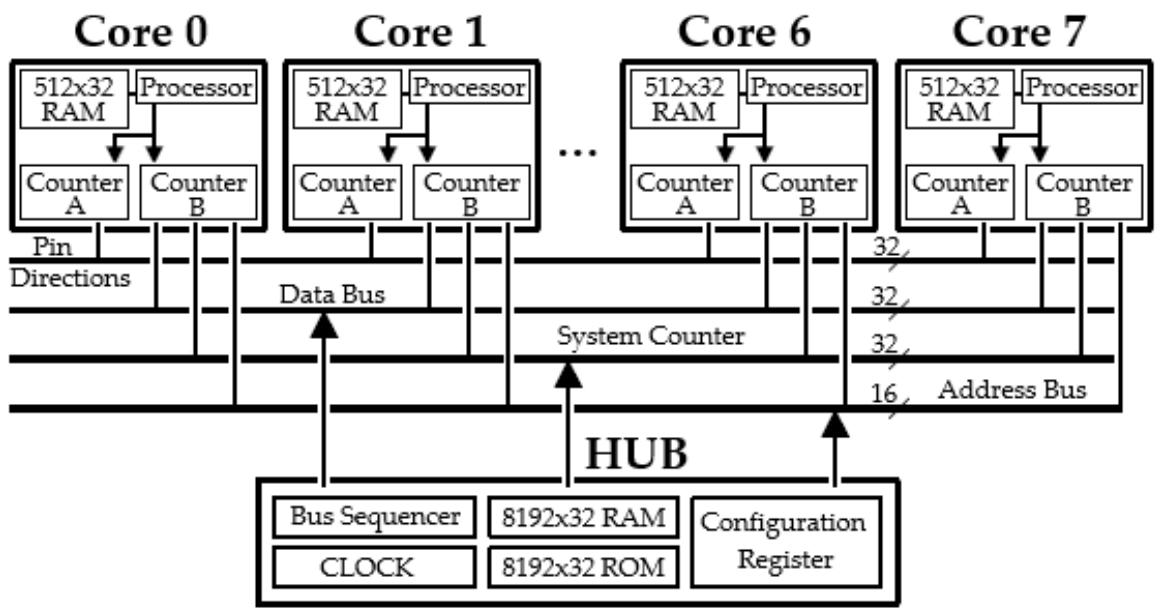

Figure 2. Propeller microcontroller block diagram.

\subsection{Signal Generation: Hardware Implementation and Control}

Figure 3 shows the block diagram of the signal generator hardware implementation. It is based on the $\mathrm{D}$ modulation technique to achieve an accurate full range $(0 \mathrm{~V}$ to $3.3 \mathrm{~V})$ quadrature signal generation needing the minimum number of external passive elements. The first quarter cycle of the two signals to be generated (sine and cosine, from 0 degree to 89.98 degrees) is stored in the shared main RAM memory of the processor. Signal points are stored using a 16-bit representation, with a maximum resolution of 4096 points per quarter. Because the main RAM memory in the processor is composed of 32-bits length registers, each memory position stores the corresponding sine (16 most significant bits-MSB of the memory position) and cosine (16 less significant bits-LSB) values, saving with this choice access time to the global memory and therefore speeding the quadrature signal generation task.

Each core sequentially accesses these data in the main memory at the microcontroller HUB by a Round Robin process schedule, which respectively feeds its two independent hardware Counter Modules, consisting of configurable state machines [25] working up to the maximum $80 \mathrm{MHz}$ clock frequency. Each counter has an adder and an accumulator, which will be employed to implement the D modulator based on pulse density modulation (PDM) using the carry bit of the adder as modulated output. By properly configuring the operation of both counters in the core, the two sinusoidal signals 
with $90^{\circ}$ phase shift required for an EIS channel can be generated with a single core. An external passive integrator converts the resultant modulated pulses into a sinusoidal signal.

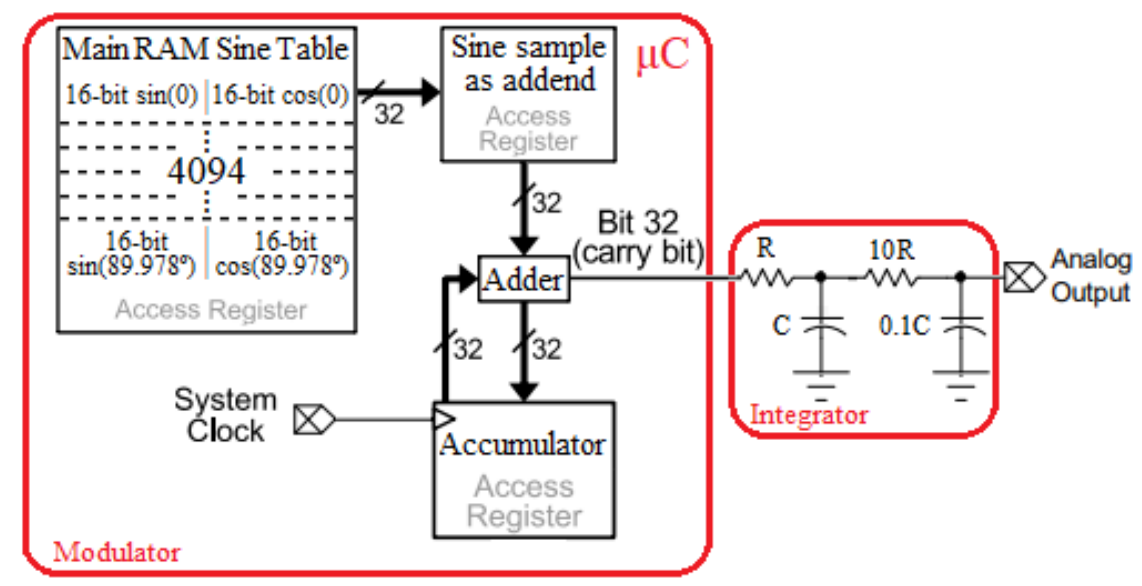

Figure 3. Signal generator hardware implementation based on D modulation.

More in detail (Figure 3), the PDM sine signal generation is performed by a core in the following manner: First, the 32-bit values representing the value of the two quadrature signals at each moment are read consecutively from the main RAM and transferred to the local memory in the selected kernel. Then, the 16 MSB bits (representing the sine value) are extracted and stored in the access register, which is in the adder, and then are added to the accumulator. When the adder overflows, the carry bit changes to 1 . The density of 1's in this output depends on the values that are being added: the higher the values accumulated, the faster the carry overflows. Thus, the density increases in the range of the maximum values of the sine function, while it decreases in the minimum values. Finally, the resulting modulated pulse train is converted into an analog signal by means of a passive second order low-pass filter (LPF) (Figure 3) consisting of two cascaded RC circuits $(\mathrm{R}=2.2 \mathrm{~kW}, \mathrm{C}=330 \mathrm{pF})$ with the same constant time and a factor of 10 in the consecutive $R^{\prime} s$ and $C^{\prime} s$ values to reduce the loading effect. The integration time is selected to keep distortion bounded below $0.75 \%$ as design specification, as will be shown next.

This process is iterated until all the values in the table are traversed. Next, the process is repeated using the LSB values. These data represent the cosine values in the first quarter of the cycle and, therefore, the sine values in the second, giving therefore signal continuity. To conclude a complete sinusoidal cycle, this process is repeated but changing the sign of the data in RAM to represent the negative half cycle. The cosine generation is performed in a similar manner.

The frequency of the sine/cosine output signal is determined by two different working frequencies: (i) The frequency of the modulated signal, which corresponds to the frequency of the square signal whose density varies. In this work, this signal matches the microcontroller clock frequency, which is set to its maximum value, $80 \mathrm{MHz}$; (ii) The data generation sampling frequency, that is, the frequency at which the system picks a sine/cosine value from the Main RAM to be sent to the Adder to provide a new output signal value. This frequency, in turn, mainly depends on the microcontroller clock frequency and the number of clock cycles required to load a value into the Adder register from the RAM, being thus more restrictive. An additional control of the output signal frequency can be achieved by selecting not all the samples, but one of every $n$ values at the RAM memory, thus reducing the number of signal points for the generation and therefore reducing the time to generate a signal period.

Taking into account all the aforementioned, the frequency of the sine/cosine output signals $f_{\text {out }}$ can be expressed as

$$
f_{\text {out }}=f_{\text {clk }} \frac{(A+1)}{2^{14}(S+D)}=f_{\text {sampling }} \frac{(A+1)}{2^{14}}
$$


being the maximum number of samples per signal period equal to $2^{14}\left(2^{12}\right.$ samples/quarter $\left.\times 4\right), A$ is the number of samples not read between two consecutive readings from the memory, $S$ is the number of clock cycles required to load a sample to the Adder register, and $D$ is an additional delay that can be added in each reading memory cycle and that serves to achieve a fine tuning in the value of the output signal frequency.

\subsection{Signal Generation: Experimental Characterization}

By using the degrees of freedom shown in Equation (1), with $f_{c l k}=80 \mathrm{MHz}, \mathrm{S}=65$, Arranging from 1 to 2000 and $D$ ranging from 0 to $2^{32}$, the frequency can be adjusted to range from $1.1 \mathrm{mHz}$ to $150 \mathrm{kHz}$ in $75 \mathrm{~Hz}$ coarse steps (given by parameter $A$ ), and fine steps given by $D$.

Figure 4 shows the PDM signal (carry bit adder pin) provided by the system, when configured to generate $f_{\text {out }}=5 \mathrm{kHz}$ and $f_{\text {out }}=150 \mathrm{kHz}$ sinusoidal output, measured by using a Tektronix ${ }^{\circledR}$ DPO4104 oscilloscope. Figure 5 shows the frequency spectrum for the $150 \mathrm{kHz}$ PDM signal $(S=65, D=0)$. The detail box shows the performance closer to the signal of interest, being the $1.080 \mathrm{MHz}$ peak, corresponding to the $\left(f_{\text {sampling }}-f_{\text {out }}\right)$ frequency, $\left(f_{\text {sampling }}=f_{\text {clk }} /(S+D)=1.23 \mathrm{MHz}\right.$, Equation (1)), the most relevant interference source. For signals generated at frequencies below 150 $\mathrm{kHz}$, this $\left(f_{\text {sampling }}-f_{\text {out }}\right)$ interference peak is kept far away enough to be irrelevant. Therefore, by properly selecting the cutoff frequency of the output LPF (Figure 3), the following sinusoidal signal is set to comply with a maximum distortion (THD and SFDR) below $0.75 \%$ over all the frequency range (Figure 6).

Figure 7 shows the generated sine signals within the operating frequency range, for $1 \mathrm{mHz}$ (Figure 7a) and $5 \mathrm{kHz}$ (Figure 7b). Figure 8 shows their corresponding spectra.

Figure 9 shows the two quadrature signals (sine-yellow and cosine-magenta) after the LPF for the upper and lower limit frequencies of the proposed generator $(150 \mathrm{kHz}$, Figure 9a and $100 \mathrm{mHz}$, Figure $9 \mathrm{~b}$ ). Note that since the counters provide both the carry signal and its negated value, a single core will give two pairs of quadrature signals, that can be used to characterize in parallel two different impedance systems, at the same frequency (signals blue and green in Figure 9a). Figure 10 shows the frequency spectrum for the $150 \mathrm{kHz}$ sine; in this case $T H D=-42.8 \mathrm{~dB}, S F D R=44.6 \mathrm{~dB}$, constituting the worst case distortion. 


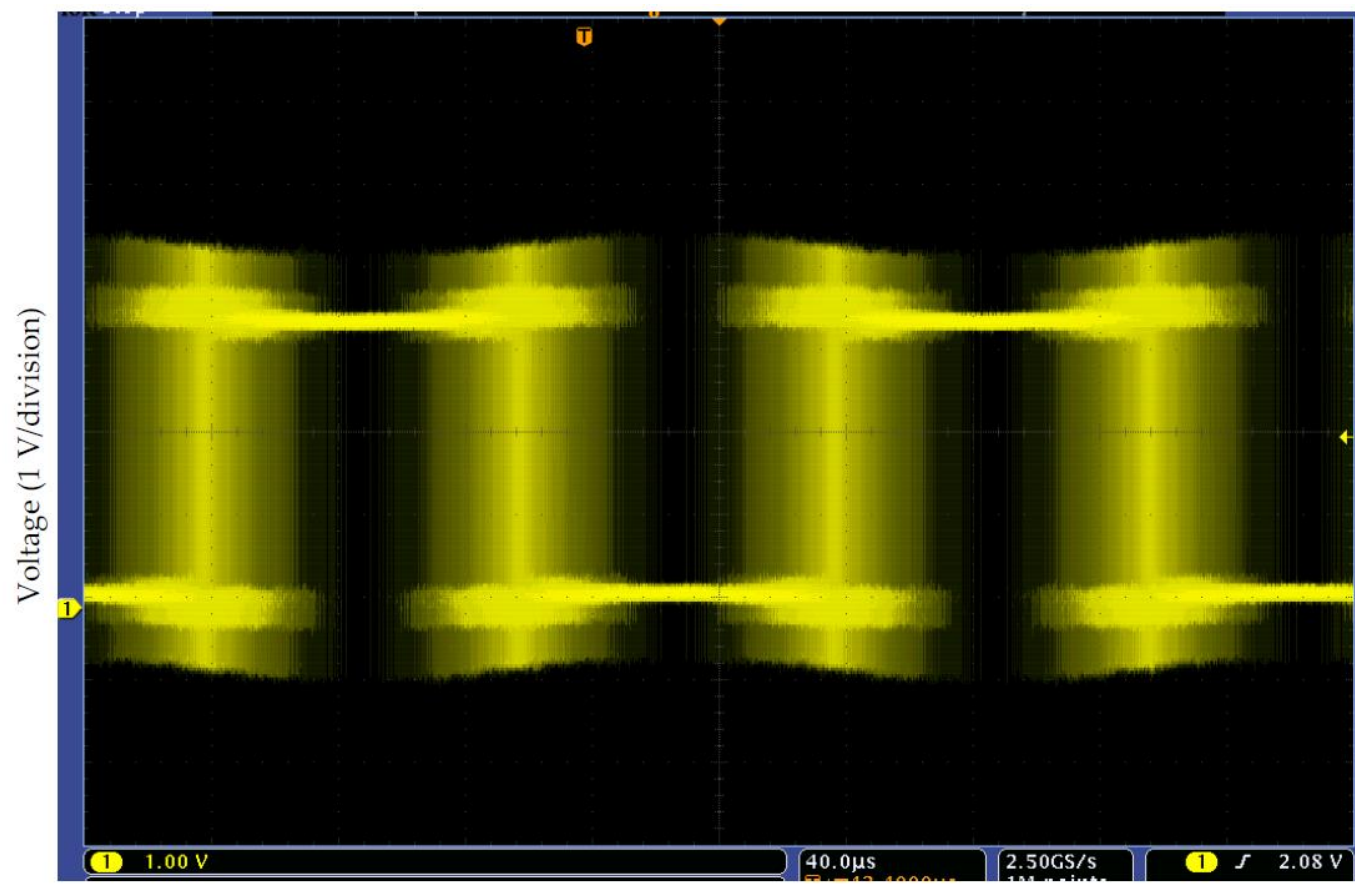

Time (40 $\mu \mathrm{s} /$ division)

(a)

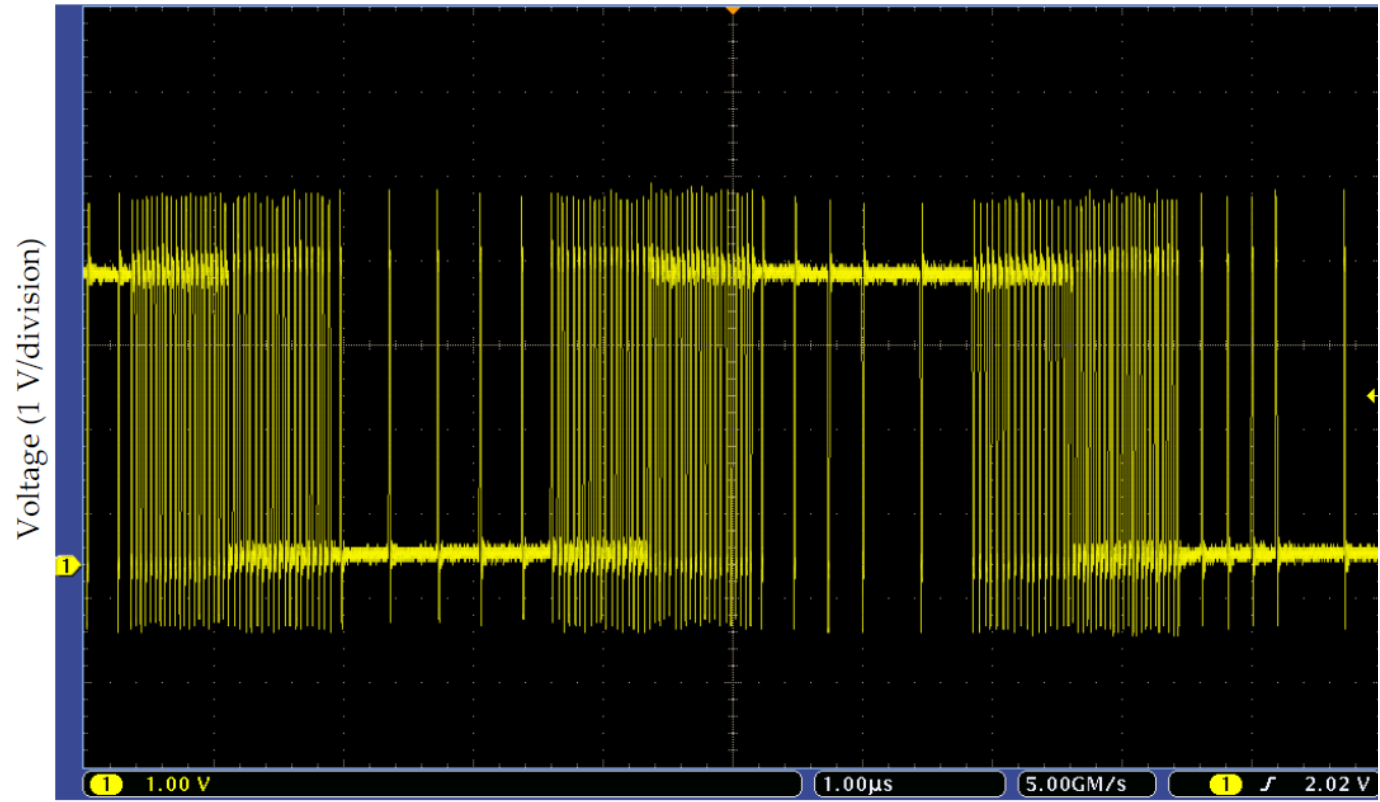

Time (1 $\mu \mathrm{s} /$ division $)$

(b)

Figure 4. Pulse density modulation (PDM) signal (voltage versus time) corresponding to (a) a $5 \mathrm{kHz}$ and (b) $150 \mathrm{kHz}$ sinusoidal outputs. The $80 \mathrm{MHz}$ pulse density is maximum for the rising and falling slopes of the sinusoidal signal and it is minimum for the maximum and minimum sinusoidal signal amplitude. 


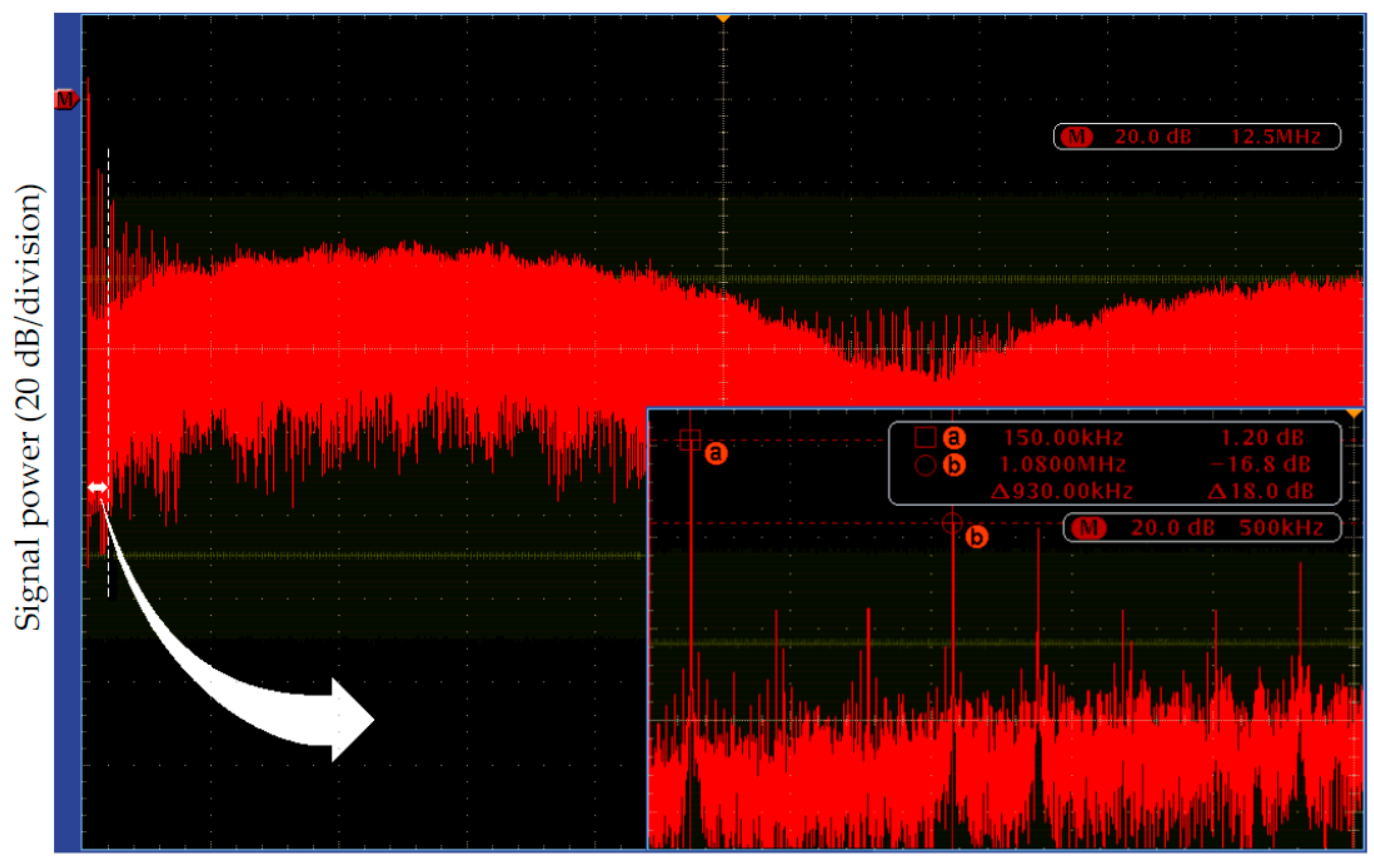

Frequency (12.5 MHz/division)

Figure 5. Spectrum (signal power in $\mathrm{dB}$ versus frequency) of the PDM signal at the carry bit output (Figure 3), before the low-pass filter (LPF) (output frequency signal of $150 \mathrm{kHz}$ ). Peak (a) corresponds to the frequency of the sine signal; peak $(\mathbf{b})$ is the $\left(f_{\text {sampling }}-f_{\text {out }}\right)$ frequency. Spectrum peaks between (a) and (b) corresponds to the 3rd and 5th harmonics of the signal. The rest of the spectrum peaks are due to the intermodal distortion. 


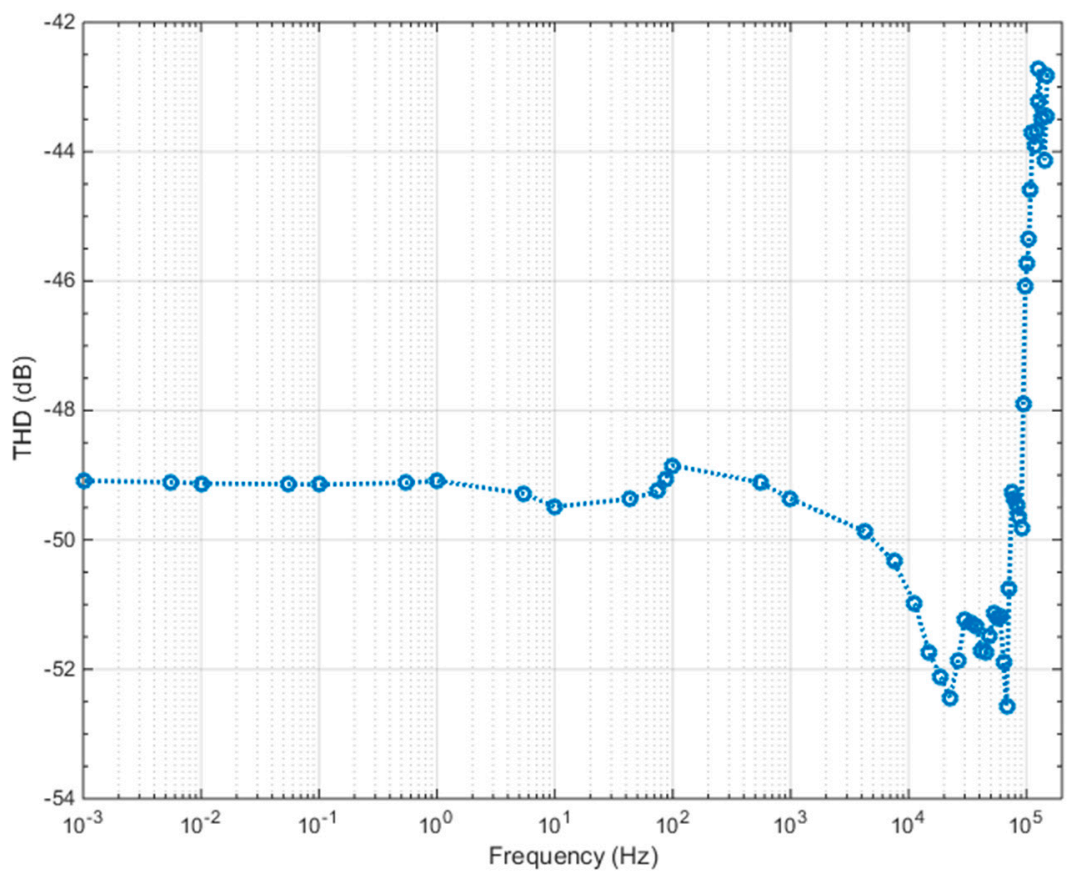

(a)

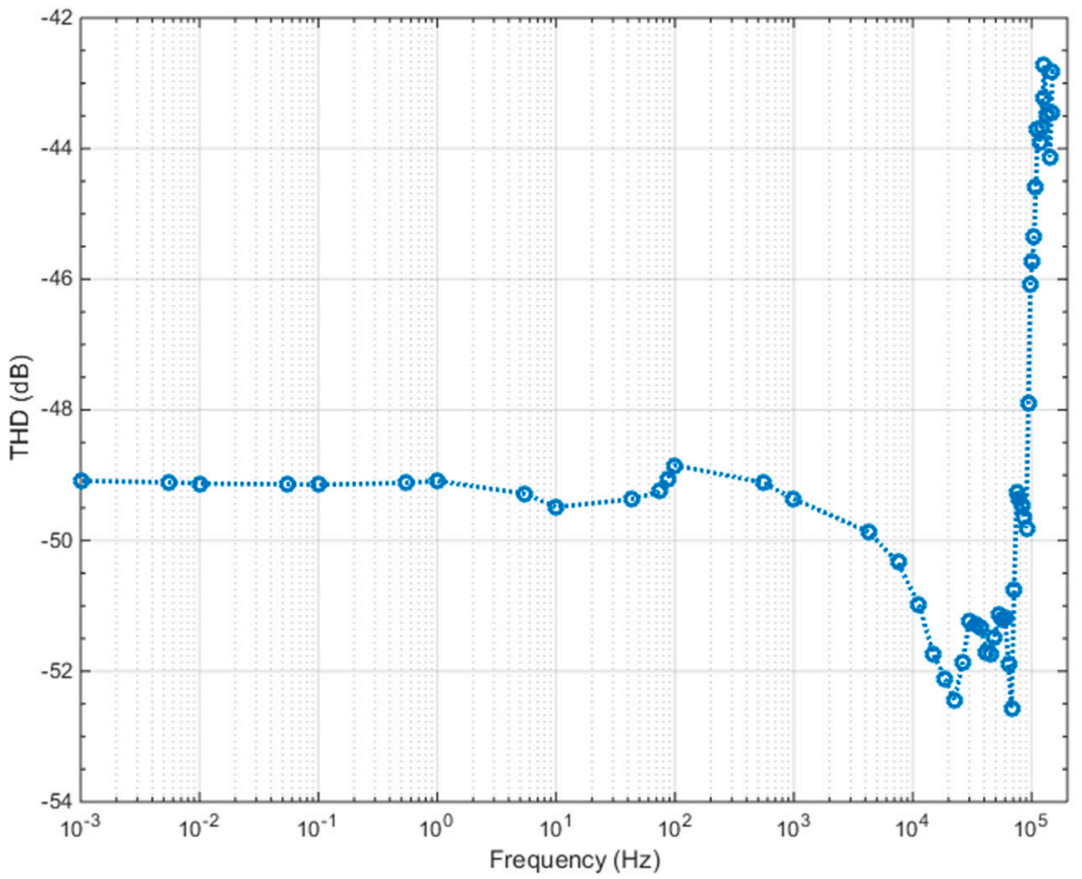

(b)

Figure 6. (a) THD and (b) SFDR experimental values for the quadrature sinusoidal signals. THD is defined as $\sqrt{\sum_{i=2}^{7} H_{i}^{2}} / H_{1}$, where $H_{1}$ is the contribution of the fundamental frequency of the sinusoidal signal, and $H_{i}$ are the successive harmonic contributions. SFDR is defined as $H_{1} / M$, where $M$ is the tone with the highest contribution which differs from $H_{1}$. Both estimators are represented in decibels. 


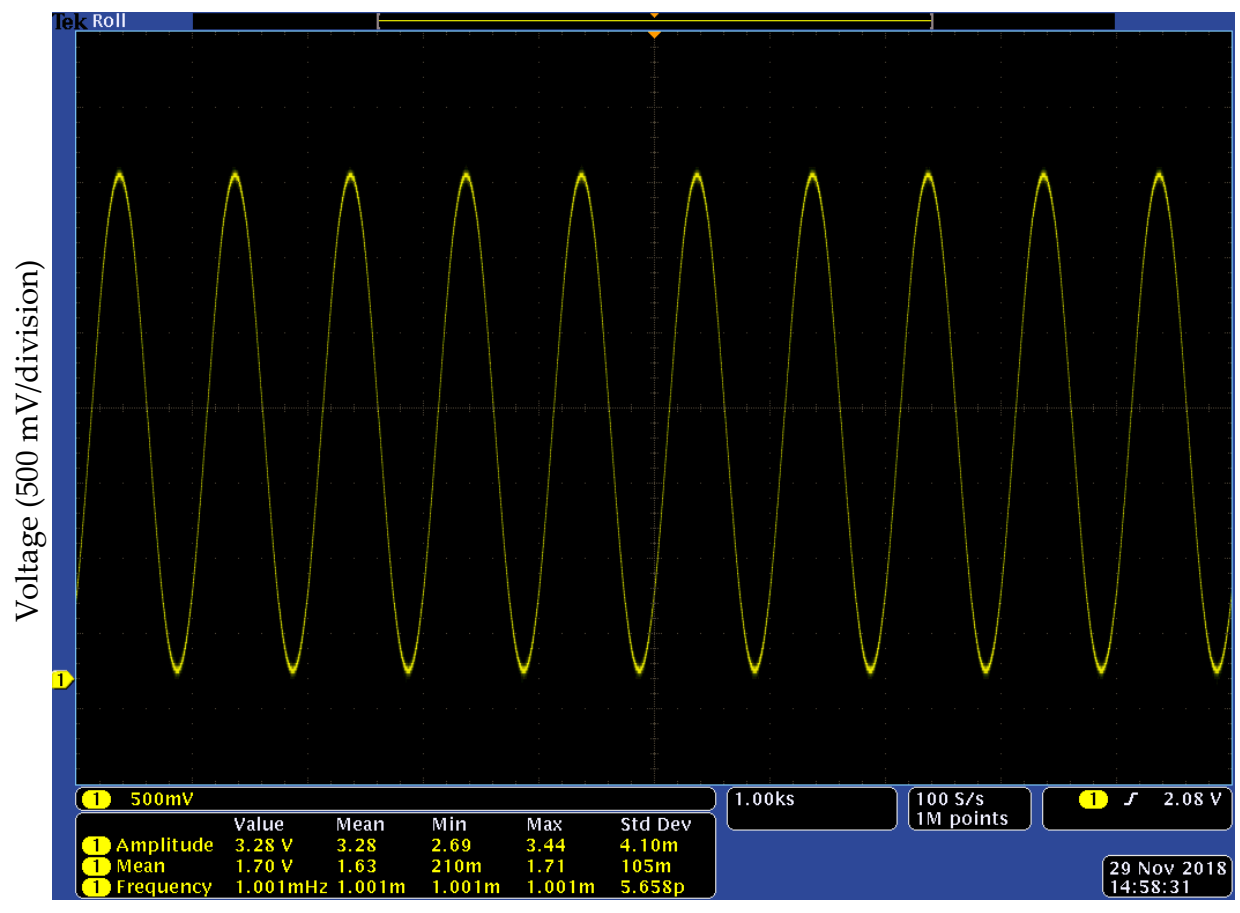

Time (1 ks/division)

(a)

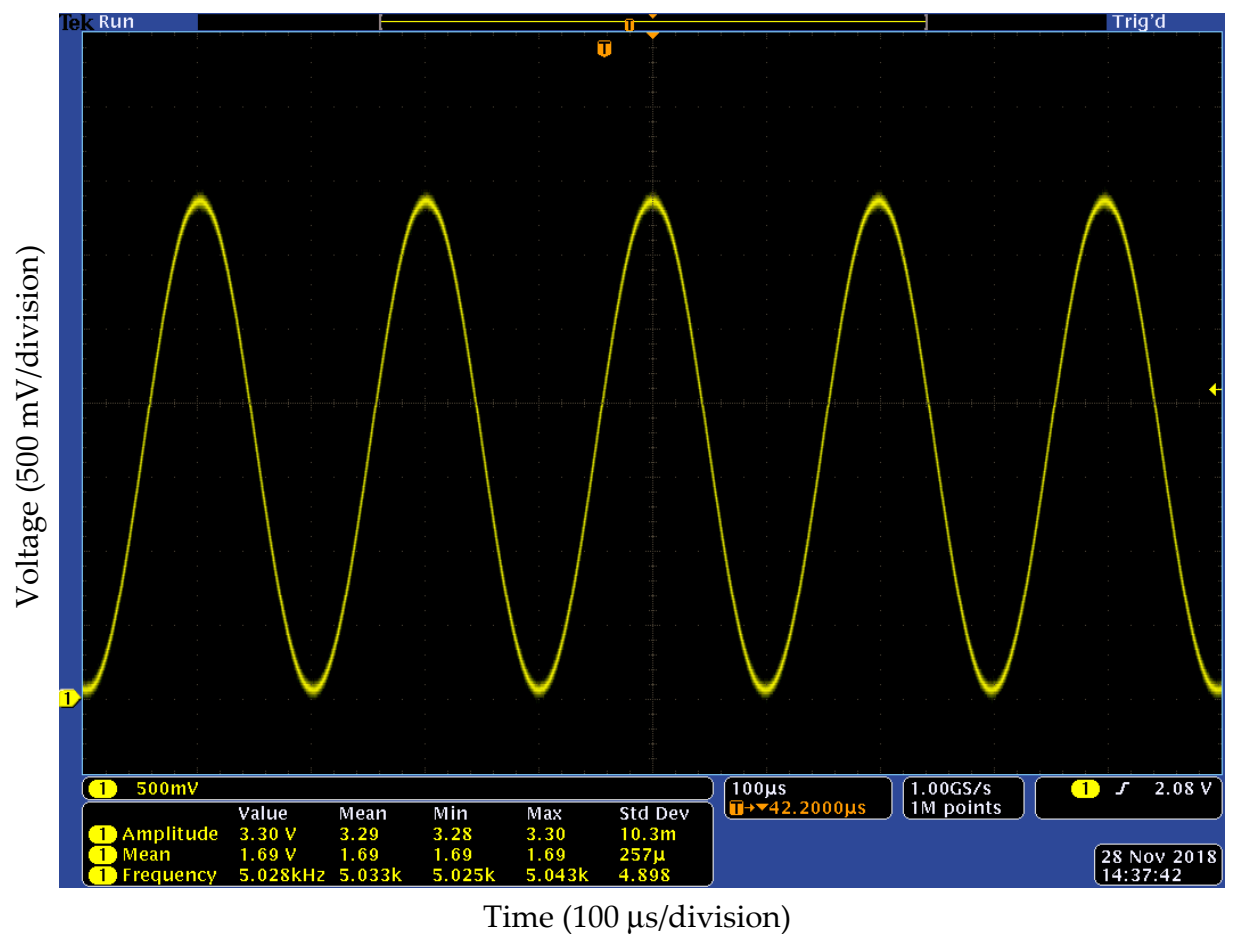

(b)

Figure 7. Sine signals (voltage versus time) generated after the corresponding PDM is low-pass filtered for the frequencies in the range of interest: (a) $1 \mathrm{mHz}$ and (b) $5 \mathrm{kHz}$. 


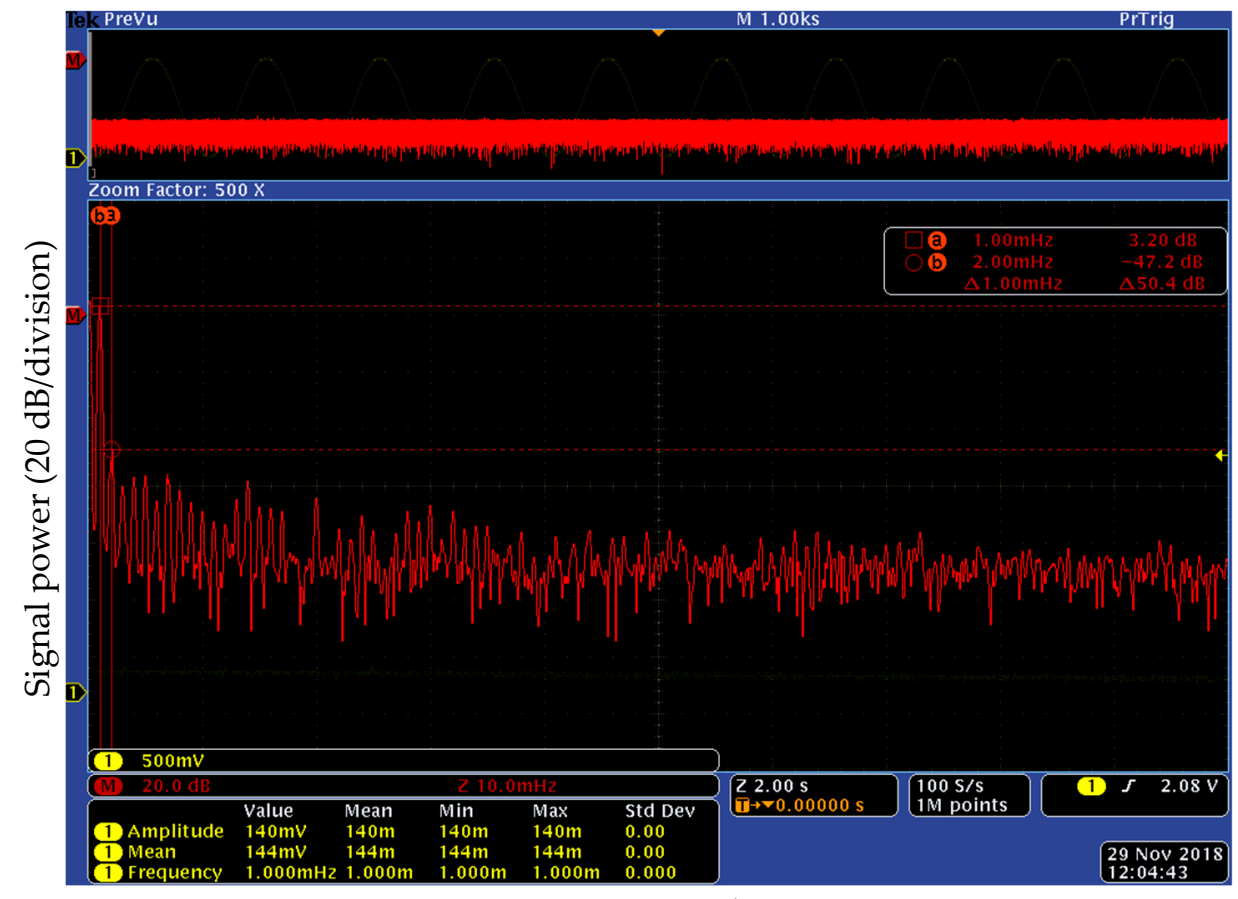

Frequency (10 $\mathrm{mHz} /$ division)

(a)

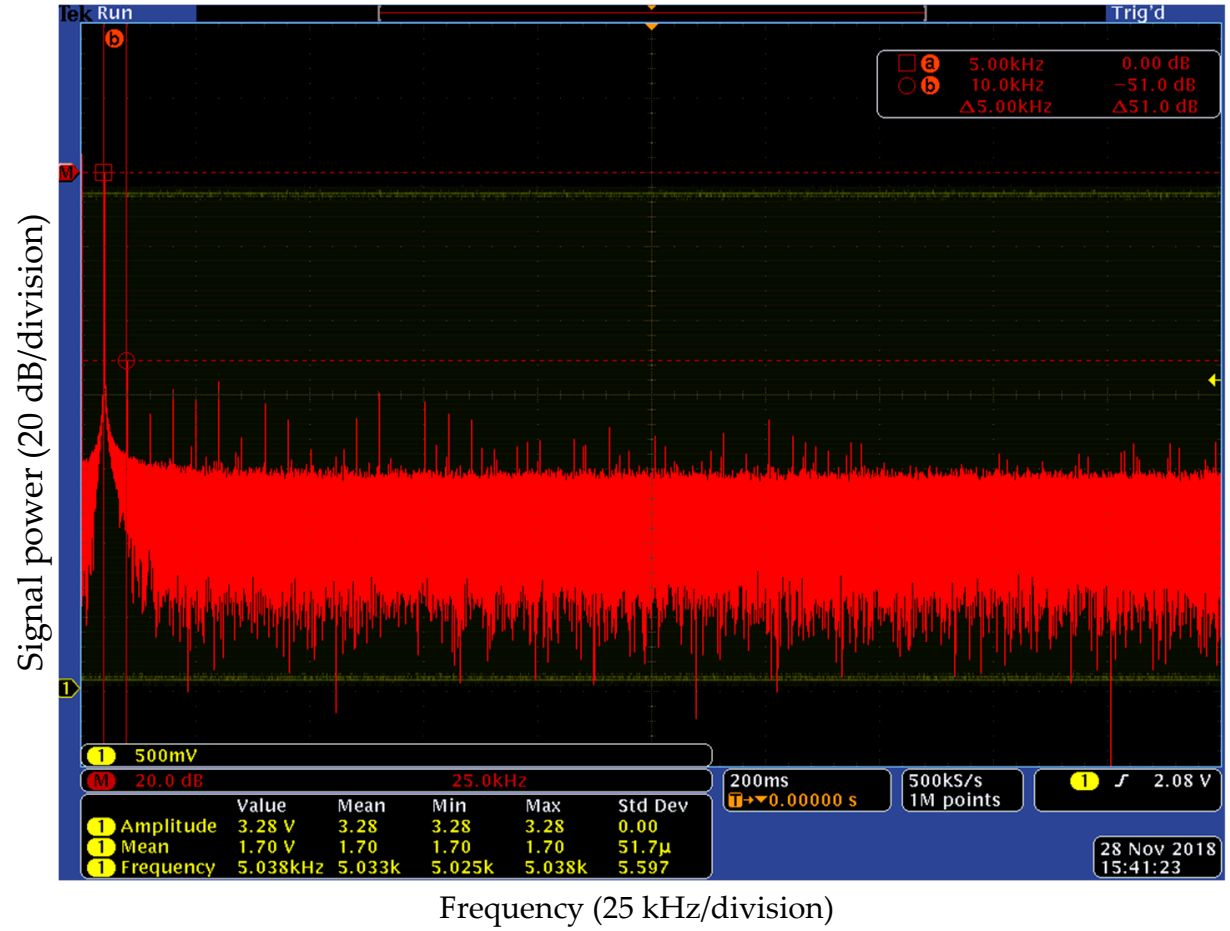

(b)

Figure 8. Spectrum (signal power in $\mathrm{dB}$ versus frequency) of the sine signals shown in Figure 7 : (a) $1 \mathrm{mHz}$ and (b) $5 \mathrm{kHz}$. 


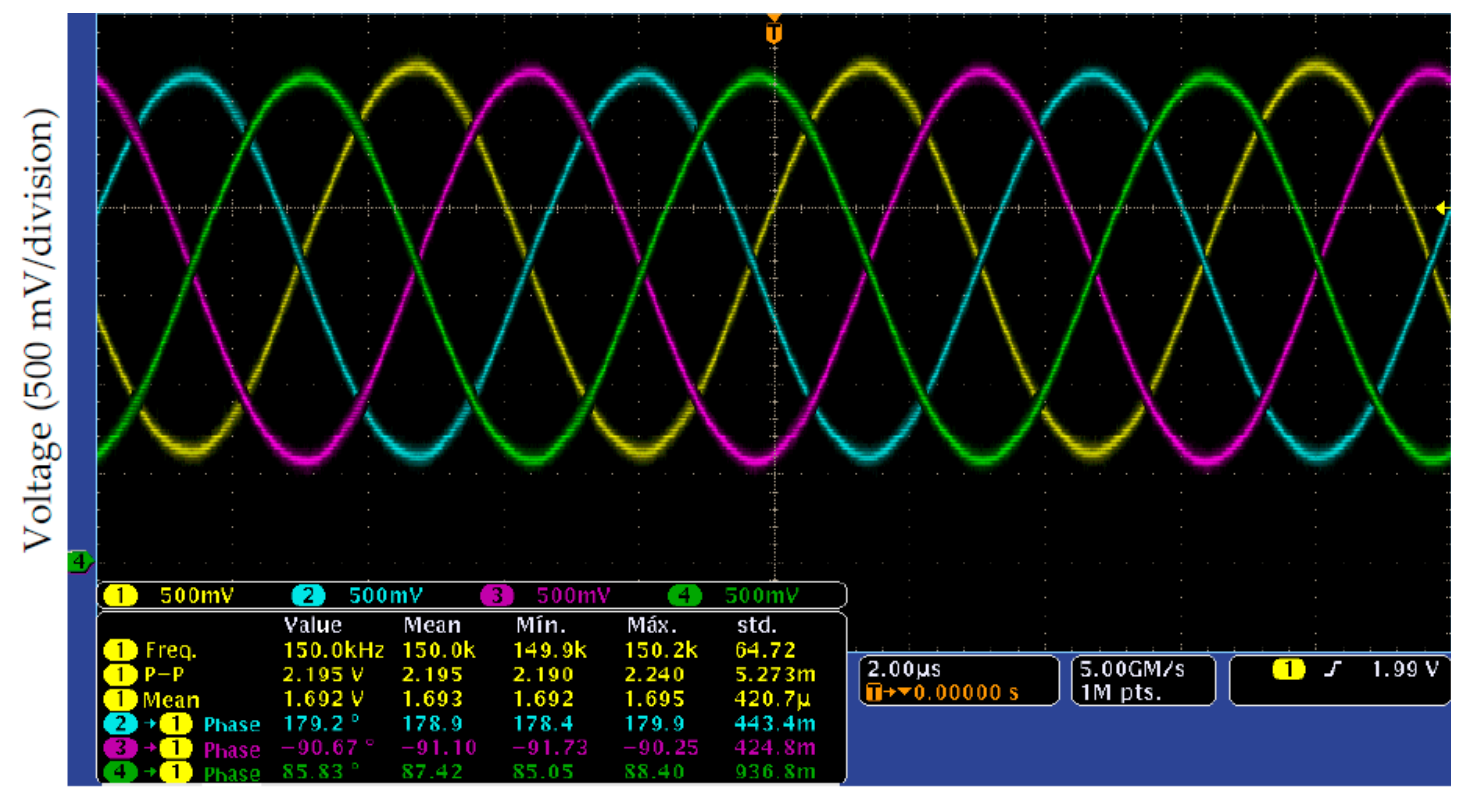

Time ( $2 \mu \mathrm{s} /$ division $)$

(a)

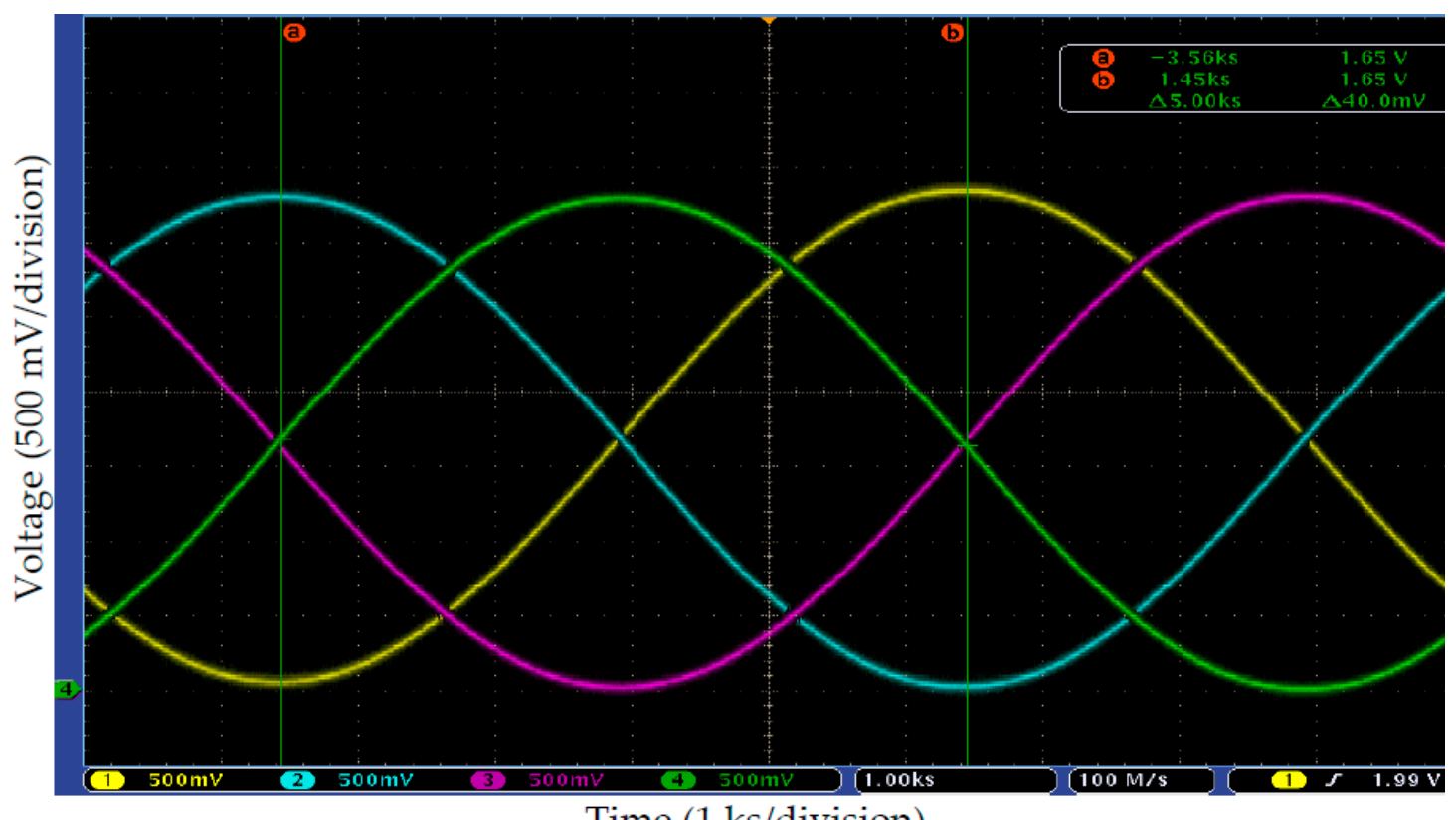

Time (1 ks/division)

(b)

Figure 9. Quadrature sinusoidal signals (voltage versus time) provided by the proposed $\Delta$ modulation Digital-to-Analog Converter (DAC) using the same LPF, for (a) $150 \mathrm{kHz}$, and (b) $100 \mathrm{mHz}$. Because the adder provides both the carry and its negated value, a core can provide two quadrature signal pairs in parallel. 


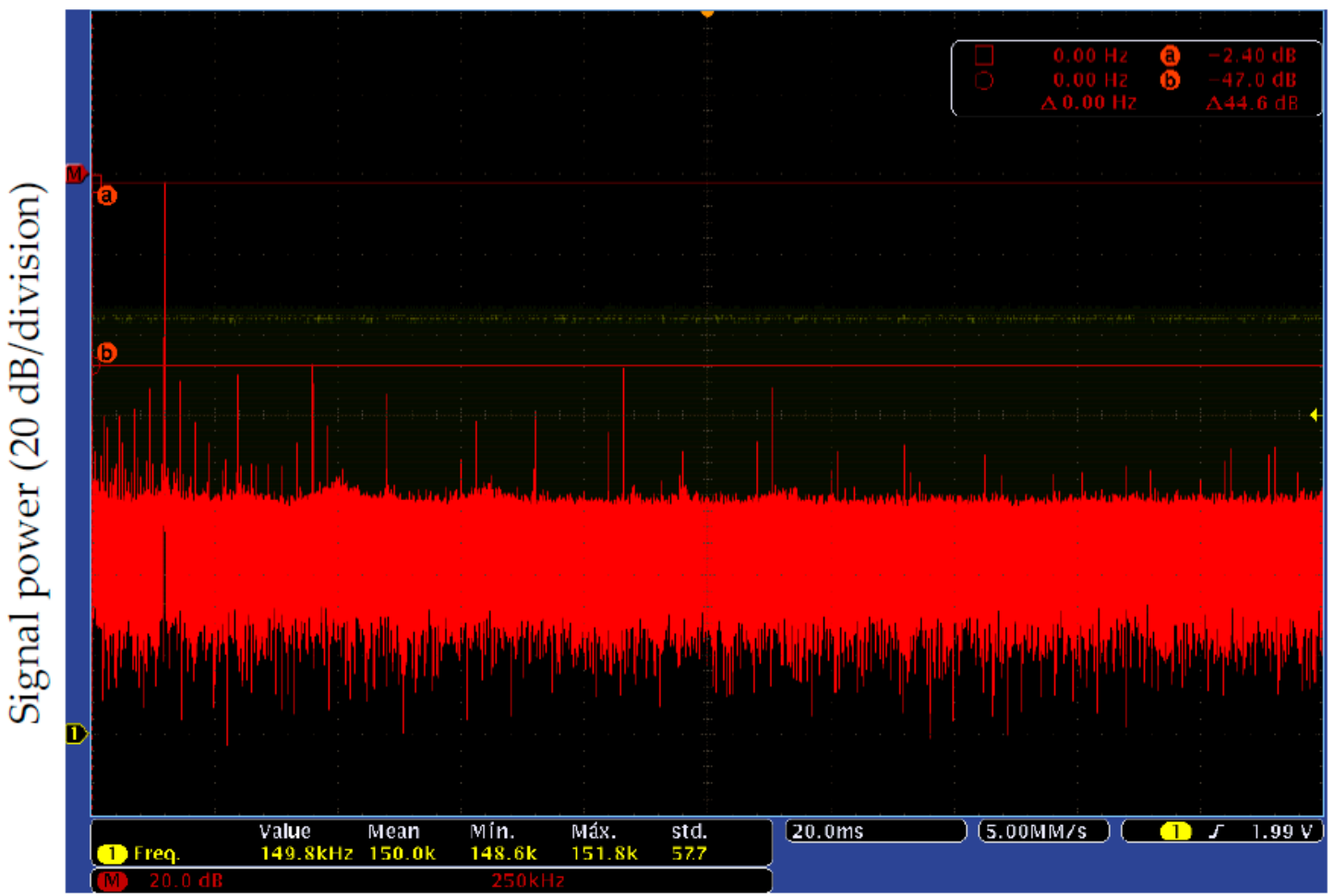

Frequency $(250 \mathrm{kHz} /$ division)

Figure 10. Spectrum (signal power in $\mathrm{dB}$ versus frequency) of the generated sine signal at 150 $\mathrm{kHz}$ frequency.

\subsection{Signal Acquisition: Hardware Implementation and Control}

The purpose of the signal acquisition stage is to recover the sensor signal to next perform the synchronous mixer operation rendering the corresponding quadrature outputs. The average of these values corresponds to the real and imaginary components or, equivalently, the magnitude and phase of the impedance under test. Note that since the average value of a signal is independent of its frequency, in all this process the signal sampling rate can be relaxed without loss of information. That is why a sigma-delta analog to digital conversion $(\Sigma \Delta-A D C)$ algorithm has been selected in spite of its low conversion rate to accomplish a more accurate conversion. In addition, the $\Sigma \Delta$-ADC main building blocks can be implemented using the internal resources of a single core, requiring minimum additional external components.

\subsubsection{Digitization}

Figure 9 shows the block diagram of the $\Sigma \Delta$-ADC. It consists on a $\Sigma \Delta$ modulator (composed by the integrator, the quantizer and 1-bit Digital-to-Analog Converter DAC blocks) plus a counter module working as a digital decimation filter [26]. Both the quantizer and the decimation filter have been implemented using the registers of the two counters available in a core. The qualitative operation of this system is as follows [27]: the analog input (Figure 11, sensor output signal), through an RC circuit formed by resistor $R_{1}$ and capacitor $C$, provides a voltage value which drives the input of a $\mathrm{D}$ flip-flop. While the voltage level in the capacitor is higher than the bi-stable threshold (assuming the threshold voltage in the bi-stable is half the digital bias voltage, $\left.V_{D D} / 2\right)$, its output $Q$ remains $' 1^{\prime}$ (and $\bar{Q}={ }^{\prime} 1^{\prime}$ ). This Output $Q$ enables the accumulator operation, increasing the value in this register for each new clock cycle. On the other hand, the output $\bar{Q}$ conforms a negative feedback loop to the input capacitor through resistor $R_{f}$ that reduces the voltage at the integrating capacitor. Once the voltage at input $\mathrm{D}$ gets under the threshold value, outputs $Q$ and $\bar{Q}$ flip their values at the next clock cycle. The accumulator stops increasing its value, providing a binary value related to the 
number of cycles that the voltage value at the analog input remains greater than the threshold value. The resolution in which the analog value is represented by the Serial Digital Out depends on the selected integration time.

In fact, the application of the circuit shown in Figure 11 to time-dependent signals presents some constrains related to the cutoff frequency of the input low-pass filter, the frequency of the input signal and the conversion frequency to guarantee a suitable estimation of the input sample. For the sake of simplicity, let us suppose the sigma-delta modulator works in linear mode (that is, the clock frequency is much higher than input signal frequency, therefore considering its operation mode as continuous). Then, the block diagram of the $\Sigma \Delta$ modulator in the $S$ domain is given by the scheme in Figure 12 [26].

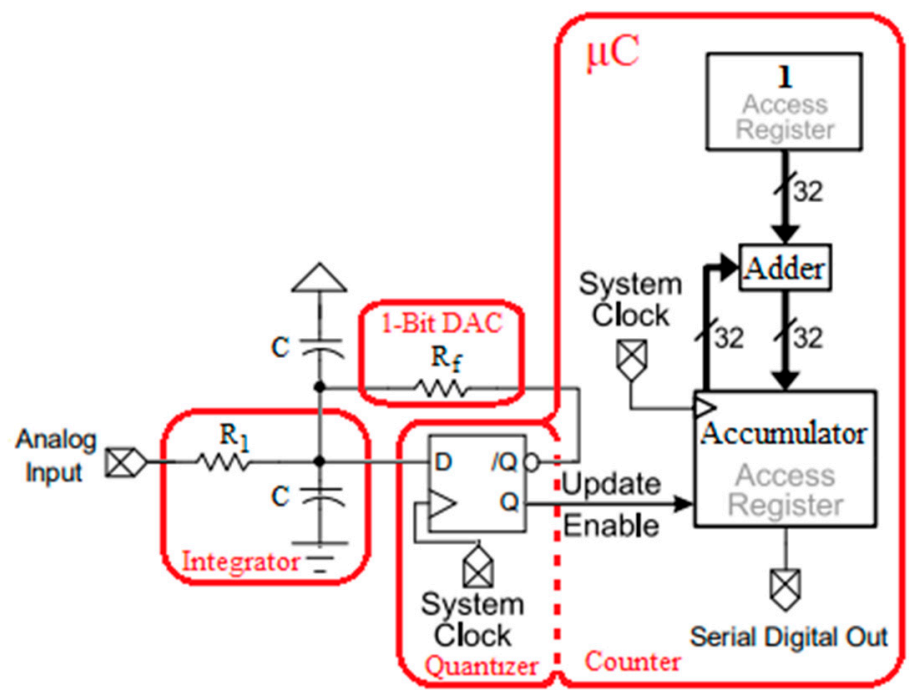

Figure 11. $\Sigma \Delta$-ADC hardware implementation.

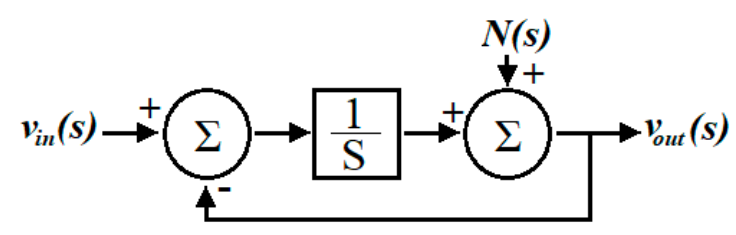

Figure 12. $\Sigma \Delta$-ADC representation in the $\mathrm{S}$ domain.

Where $v_{\text {in }}(s)$ is the input voltage and $v_{\text {out }}(s)$ is the output $Q$ in Figure 11, while $N(s)$ represents the effect of quantization in the transfer function, which is negligible if linear operation is assumed. The $\Sigma \Delta$ modulator transfer function is

$$
\frac{v_{\text {out }}(s)}{v_{\text {in }}(s)}=\frac{1}{1+s}
$$

and accordingly, the output voltage can be expressed as

$$
v_{\text {out }}=\frac{R_{f}}{R_{1}} \frac{1}{1+2 s R_{1} C} v_{\text {in }}+K
$$

where $\mathrm{K}$ is proportional to the $\mathrm{D}$ flip-flop threshold voltage, $V_{D D} / 2$. Therefore, the output voltage depends on the $f_{R_{1} C}$ passive filter cutoff frequency and the input signal frequency $f_{\text {in }}$. Thus, an input signal with a frequency higher than $f_{R_{1} C}$ may result in a loss of accuracy. Besides, the conversion 
frequency $f_{\text {conv }}$ must be fast enough to avoid that the capacitor discharge process affects the digitized value, which would reduce the resolution in bits of the ADC. That is, on the overall it must be satisfied

$$
f_{\text {in }} \leq f_{R_{1} C} \leq f_{\text {conv }} \approx \frac{f_{c l k}}{2^{N}}
$$

being necessary to appropriately select the passive components in the modulator stage as well as the conversion rate in order to perform a suitable signal digitization in $\mathrm{N}$ bits.

To manage the hardware resources to reliably perform the required operations while minimizing the execution time, a specific code using the microcontroller assembler has been developed. Figure 13 shows the simplified control flowchart describing the signal digitization and data acquisition. After configuring the corresponding input and output pins and the counter register, first a calibration process is carried out. This task, which is performed at the system start up, allows determine both the offset at $0 \mathrm{~V}$ in the accumulator and the integration time required to properly acquire the maximum allowable input voltage, therefore maximizing the dynamic range. For completing the calibration step, the Analog Input (Figure 11) is connected to $0 \mathrm{~V}$. After the integration time, the value stored in the accumulator, which ideally should be equal to zero, is the excess offset reading that must be subtracted from the system readings in normal operation mode. The integration time is adjusted by applying the maximum voltage to be digitized in the Analog Input. After the integration time, the accumulator should be filled to the maximum value $\left(2^{N}\right)$, keeping the overflow flag equal to zero. Otherwise, the integration time must be increased/decreased up to reach this condition for a given number $\mathrm{N}$ of bits.

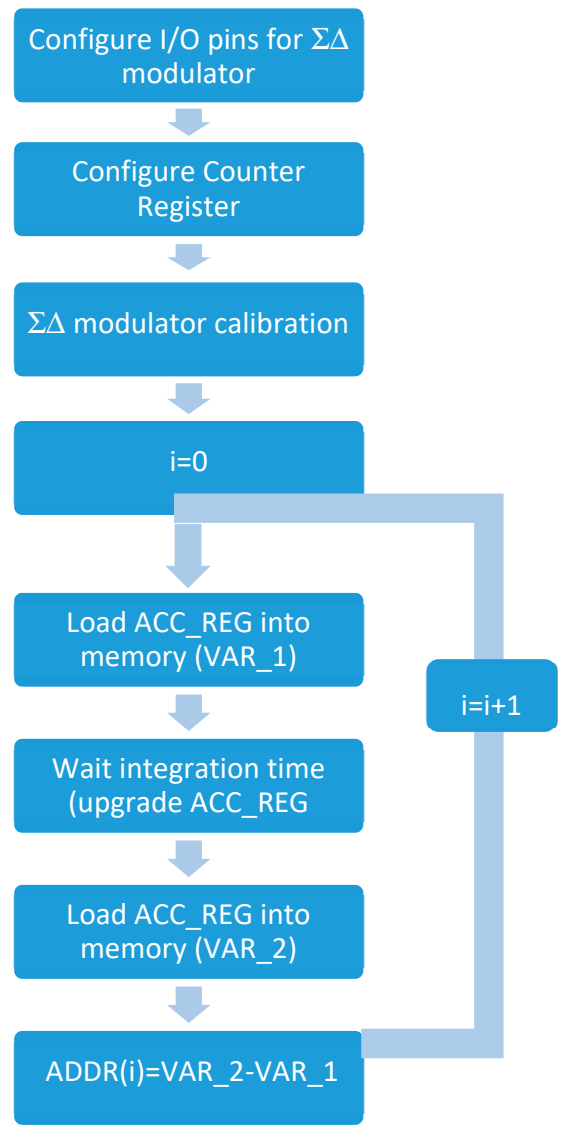

Figure 13. $\Sigma \Delta$-ADC control flowchart.

Once calibrated, the system temporally saves the value stored in the counter accumulator in a variable, waits the integration time and reads again the value in the register. The difference between 
both data represents the value of the sensor output signal at this time, which is stored in a memory address so that data from consecutive instants of time use consecutive memory positions.

\subsubsection{Mixing and Averaging}

In a Propeller microcontroller, the hardware resources allow digitizing up to two different signals in parallel per core. To accurately synchronize the mixers operation, the system makes use of a dedicated core for each impedance measurement according to the following process: one of the counters is dedicated to digitize the signal arriving from the impedance under study, while the other counter synchronously digitizes the original sinusoidal excitation signal. The product of these two data corresponds to the real mixer output. The real component of the impedance under test is then calculated by averaging the products provided by this branch over a minimum $n=5$ periods of the excitation signal to obtain reliable results over all the operating frequency range. In a single-channel measurement approach, the quadrature signal, which does not excite the impedance under study (cosine) is directly read from the hub memory, feeding the corresponding mixer (Imaginary) before its averaging, thus making unnecessary its digitization, which saves both hardware as computing resources.

\subsection{Signal Acquisition: Experimental Characterization}

The structure shown in Figure 11 has been implemented for a 12-bit approach. First, the linearity of the analog-to-digital conversion is verified by applying an incremental DC voltage in the biasing range of the microcontroller (from 0 to $3.3 \mathrm{~V}$ ) to the Analog Input of the $\Sigma \Delta$-ADC (Figure 11), and recovering the output digital values. Figure 14 shows the results, where y axis corresponds to the analog values represented by the digital words obtained in the conversion, assuming a full scale digitization (that is, $000 \mathrm{~h}$ represents $0 \mathrm{~V}$ and FFFh represents $3.3 \mathrm{~V}$ in hexadecimal). It can be seen in this figure that the ADC conversion presents high linearity, resulting in a gain or slope of 0.65 , an offset below $18 \mathrm{mV}$, and with a coefficient of determination $\bar{R}^{2}=1.0000$. The conversion slope can be modified by the feedback loop through $\frac{R_{f}}{R_{1}}$ (Equation (3)), to adjust its value according to the conversion requirements. In this case, to allow a full sweep in the supply voltage range avoiding saturation in the digitization module (Figure 11), we kept the output gain $<1$ by selecting $R_{f}=100 \mathrm{k} \Omega$ and $R_{1}=155 \mathrm{k} \Omega$. This choice results in a conservative 0.65 gain, exactly as obtained from the linear fit.

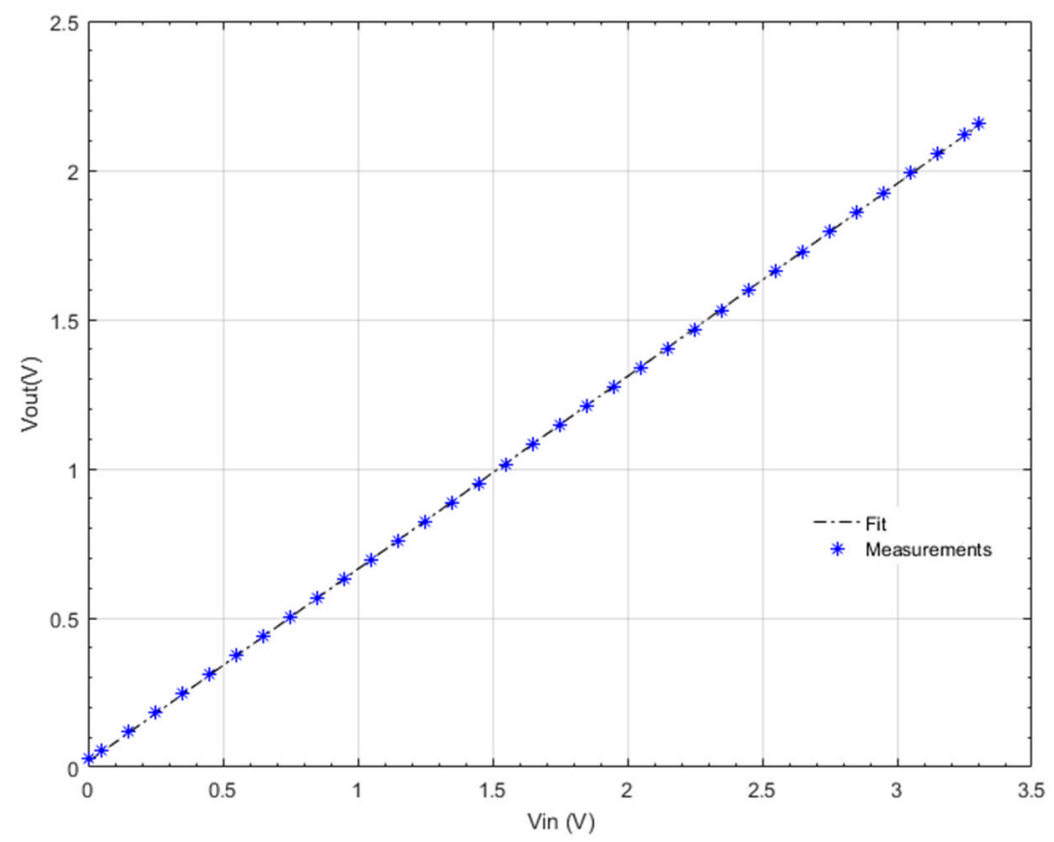

Figure 14. $\Sigma \Delta$-ADC output linearity. 
Figure 15 shows the gain versus frequency characteristic of the proposed ADC configuration, for 10-bit (red dots) and 12-bit (green dots) output resolution, which presents the typical sinc digital filter shape, matching with the previous DC characterization. According to this figure, a 12-bit ADC is selected, to enhance resolution and preserving the frequency of operation up to the $10 \mathrm{kHz}$ range.

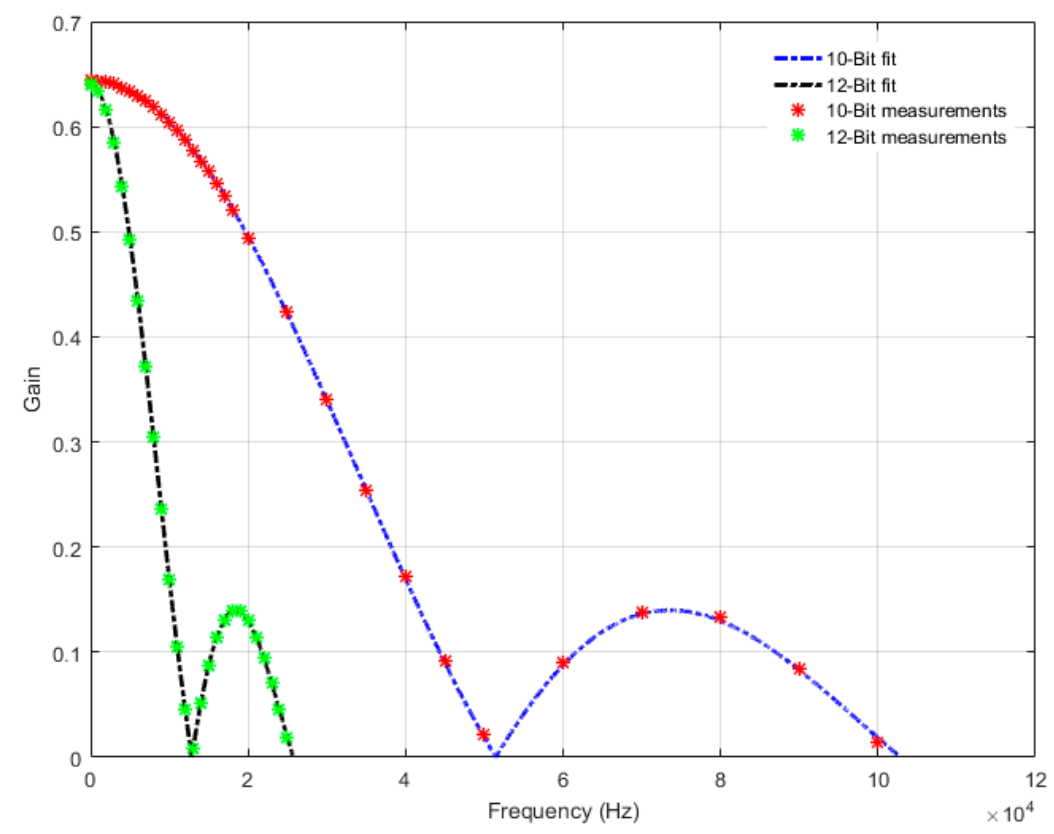

Figure 15. $\Sigma \Delta$-ADC gain response.

Finally, Figure 16 presents the voltage values acquired using the ADC (red dots) from a $5 \mathrm{kHz}$ sine signal, and the corresponding cosine values (recovered from the RAM memory using the instant sine values acquired). Both show a good matching when represented over their corresponding original full signals. These values are the inputs for the mixing operation.

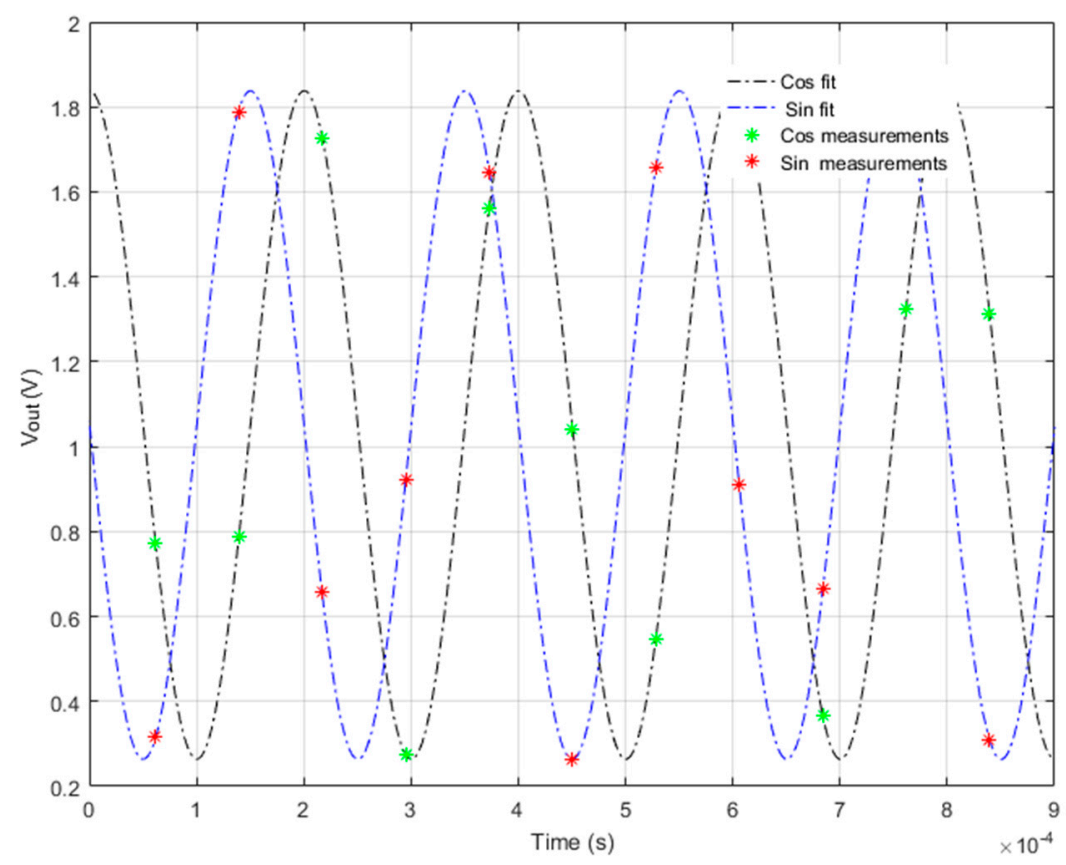

Figure 16. Signal acquisition using the proposed ADC for a sine voltage (red dots). Cosine signal is digitally recovered from the Main Memory using the sine values. 


\section{Results}

The EIS system schematic, considering a two-channel measurement approach, is shown in Figure 17, and the prototype photograph is shown in Figure 18. In Figure 17, Block (a) corresponds to the signal generation module, including the digital Counter Module in the corresponding core and the R-C low-pass filter $\left(R_{\Delta}=2.2 \mathrm{k} \Omega ; C_{\Delta}=330 \mathrm{pF}\right)$. It is followed by an impedance adapter element (a simple voltage follower), which allows transferring the generated signal to the cell under test. For real biological applications, this module can be replaced by voltage or current reduction modules suited for the target application. The Randles cell representing the impedance sample corresponds to Block (b). Note that each Randles cell is followed by a transimpedance amplifier (TIA) consisting on an Operational Amplifier with a feedback resistor $R_{f 2}$, that converts the current $I_{Z}$ provided by the biocell into a voltage value $V_{Z}=-R_{f 2} I_{Z}$ for its digitization. Block (c) represents the implemented $\Sigma \triangle$-ADC -based system that conforms the synchronously digitized impedance extraction; the passive components values are $\left(R_{f 1}=100 \mathrm{k} \Omega ; R_{\Sigma \Delta}=150 \mathrm{k} \Omega ; R_{C}=4.7 \mathrm{k} \Omega ; C_{\Sigma \Delta}=250 \mathrm{pF}\right)$, where $R_{C}+R_{\mathrm{SD}}$ corresponds to $R_{1}$ in Figure 11. This configuration allows calibrate the operation of the ADC (Figure 17) by setting the voltage value in (A) at the required values through the $\Sigma \Delta$ calibration pin without the need of deactivating the operation of the cell, thus with minimum waste of time.

\section{Application to Impedance Spectroscopy}

The system operation as a frequency response analyzer applied to impedance spectroscopy has been tested using the characteristic impedance of a biological model based on the bilayer lipid membrane presented in [28,29] (Figure 19). The associated Randles cell is modeled using three impedances whose values are: $R_{m}=434 \mathrm{~kW}, C_{m}=580 \mathrm{nF}$ and $C_{d l}=340 \mathrm{nF}$ (Figure 17). The resistor $R_{s}$ represents the impedance associated to the sensing electrodes, which can vary from negligible values up to a few MW. In this work, an intermediate value of $500 \mathrm{~kW}$ has been selected. The TIA active block in Figure 17, Block (c) is a MAX4231, and resistor $R_{f 2}=500 \mathrm{~kW}$ to accommodate a full analog voltage input range of 0 to $V_{D D}$.

For a normalized amplitude excitation signal with operating frequency $f_{i n}$, the corresponding output biosensor signal $V_{Z}$ is given by

$$
V_{Z}=-\frac{R_{f_{2}}}{|Z|} \sin \left(\omega_{i n} t+\theta\right)
$$

where $Z$ represents the cell impedance.

The digitized values of the biosensor signal are multiplied in the corresponding microcontroller core by the respective digital sine and cosine values, and the results are averaged over an integer number $n$ of signal periods (with $n$ minimum $=5$ as pointed in Section 2.3.2), so that:

$$
R e=-\frac{R_{f_{2}}}{2|Z|} \cos \theta^{\wedge} I m=-\frac{R_{f_{2}}}{2|Z|} \sin \theta
$$

Note that since each digitization at the $\Sigma \Delta$-ADC requires a minimum of $2^{12}$ clock cycles (for a 12-bit resolution), the two mixing and accumulation cycles are performed in real time by computational resources in the same core. Thus, impedance magnitude and phase shift can be recovered as

$$
|Z|=-\frac{R_{f_{2}}}{2 \sqrt{R e^{2}+I^{2}}}{ }^{\wedge} \theta=\tan ^{-1}\left(\frac{I m}{R e}\right)
$$

The impedance characterization has been performed for 18 frequency values in the $100 \mathrm{mHz}$ to $10 \mathrm{kHz}$ range at 12-bit resolution. Since for each of these measurements an acquisition time of at least 5 signal cycles has been guaranteed, a total time of $100 \mathrm{~s}$ is required for the complete characterization over frequency. Figure 20a shows the impedance magnitude recovery performance compared to the 
ideal values, while Figure 20b presents the phase evolution. Figure 21 shows the impedance magnitude and phase relative error achieved estimating both values.

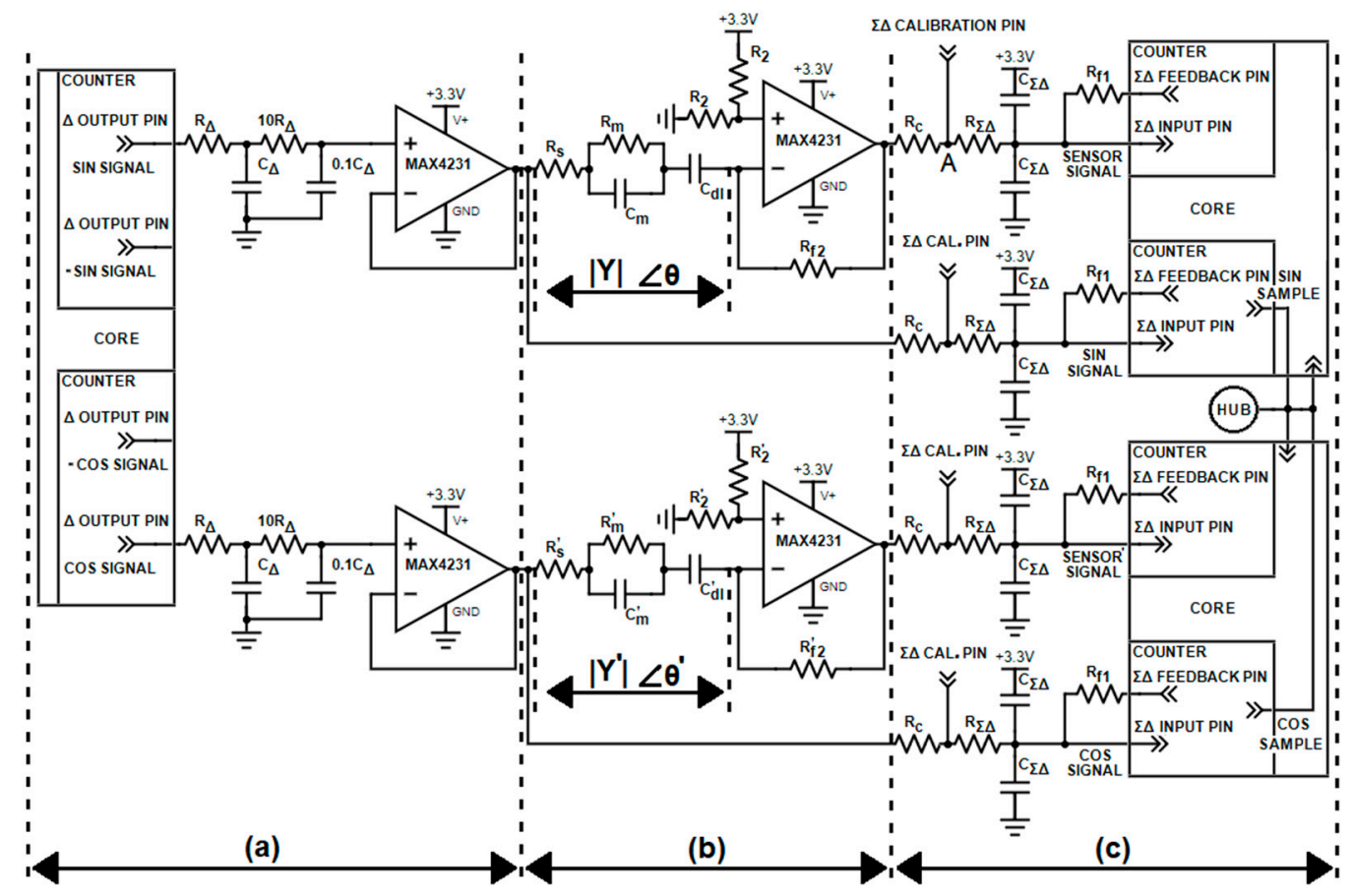

Figure 17. Block diagram for a two-channel Frequency Response Analyzer (FRA) using a single core as signal generator.

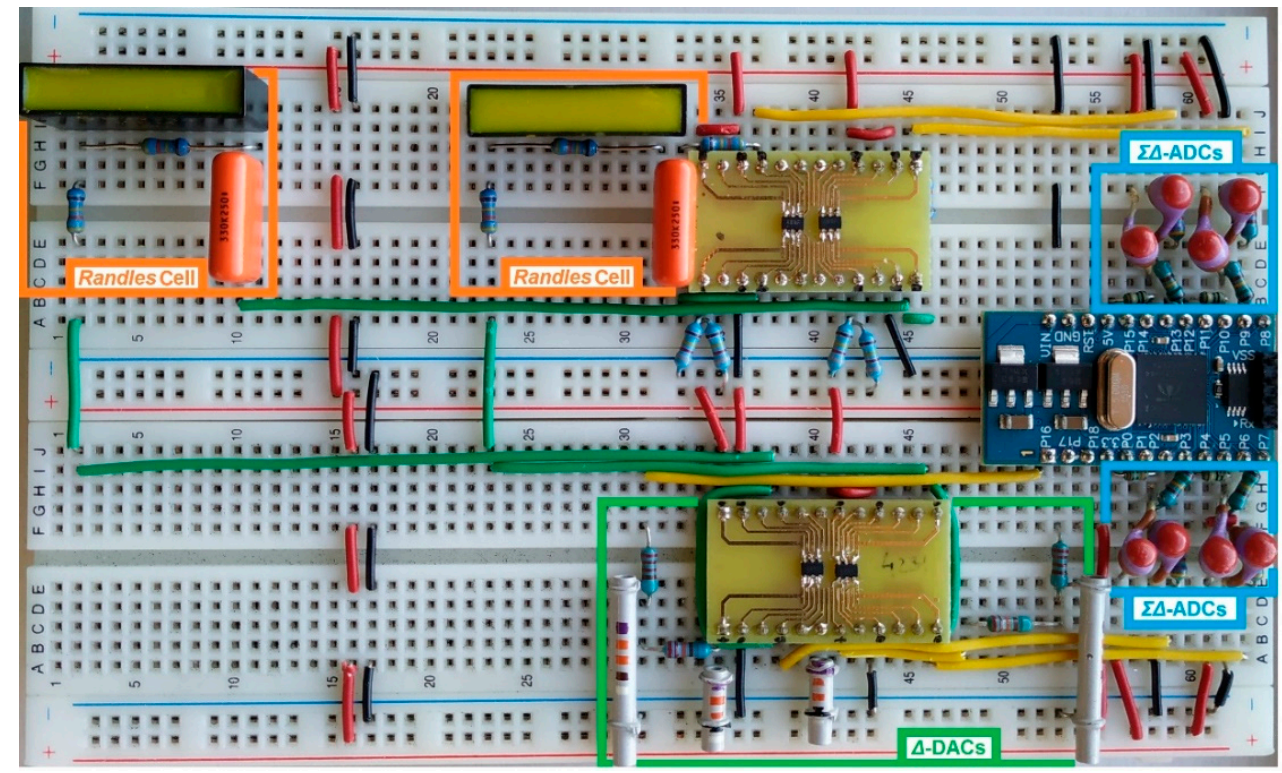

Figure 18. Two-channel prototype photograph. 


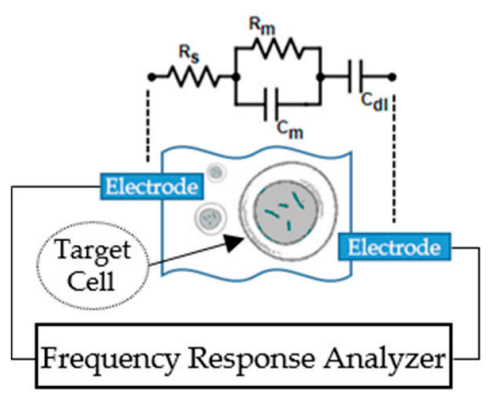

Figure 19. Application example of the proposed FRA system. The bilayer lipid membrane is characterized by applying a frequency-variable signal and measuring its response, using the proposed microcontroller device.

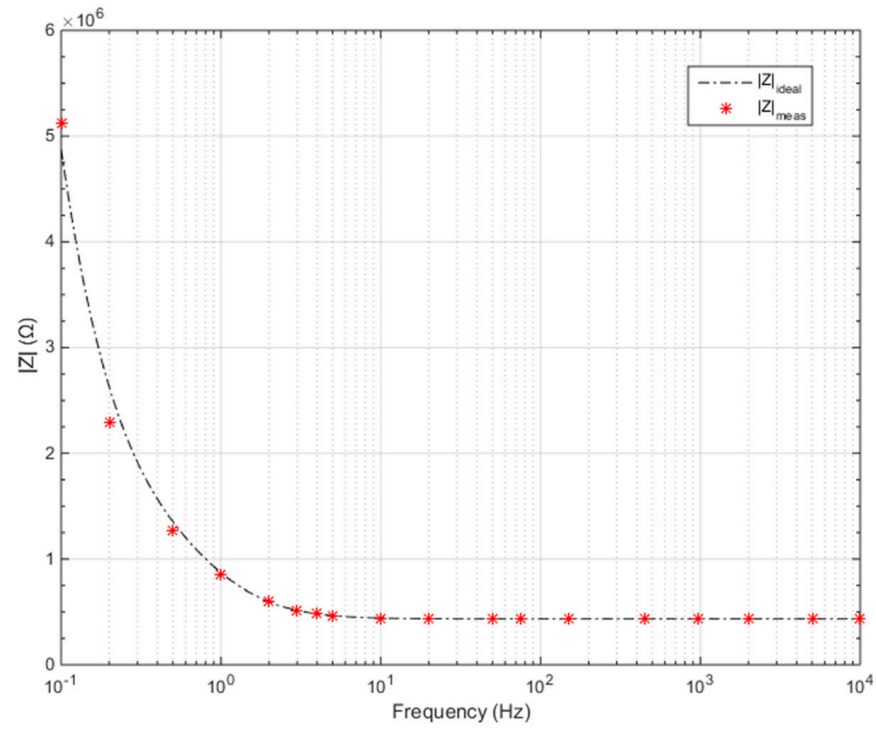

(a)

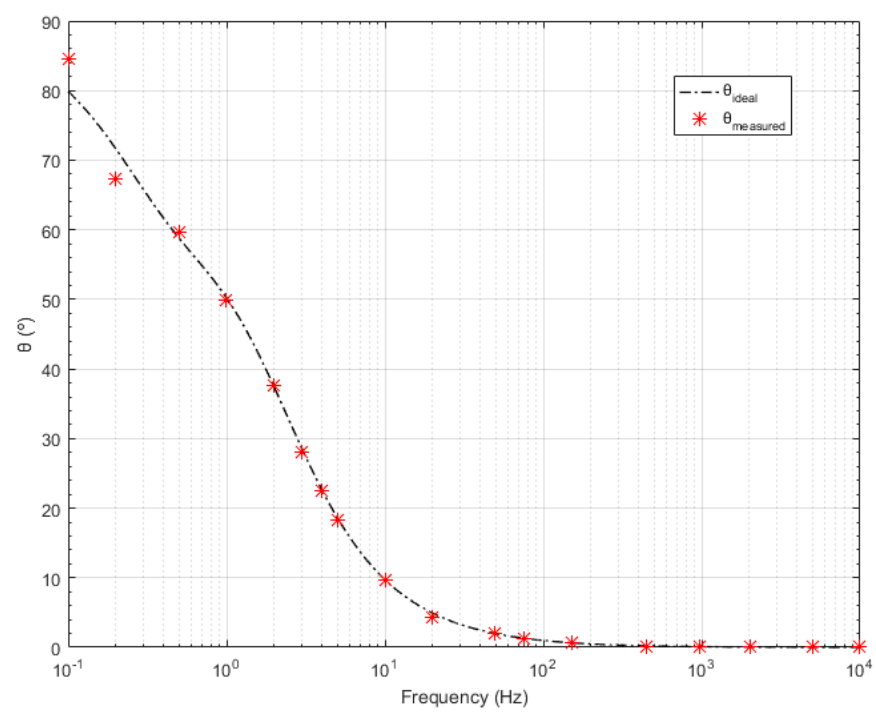

(b)

Figure 20. (a) Impedance magnitude recovery for 18 different frequencies in the $100 \mathrm{mHz}$ to $10 \mathrm{kHz}$ range. Red dots represent the experimental values recovered, dashed line is the ideal impedance value. (b) Recovered (red dots) phase values and ideal behavior. 


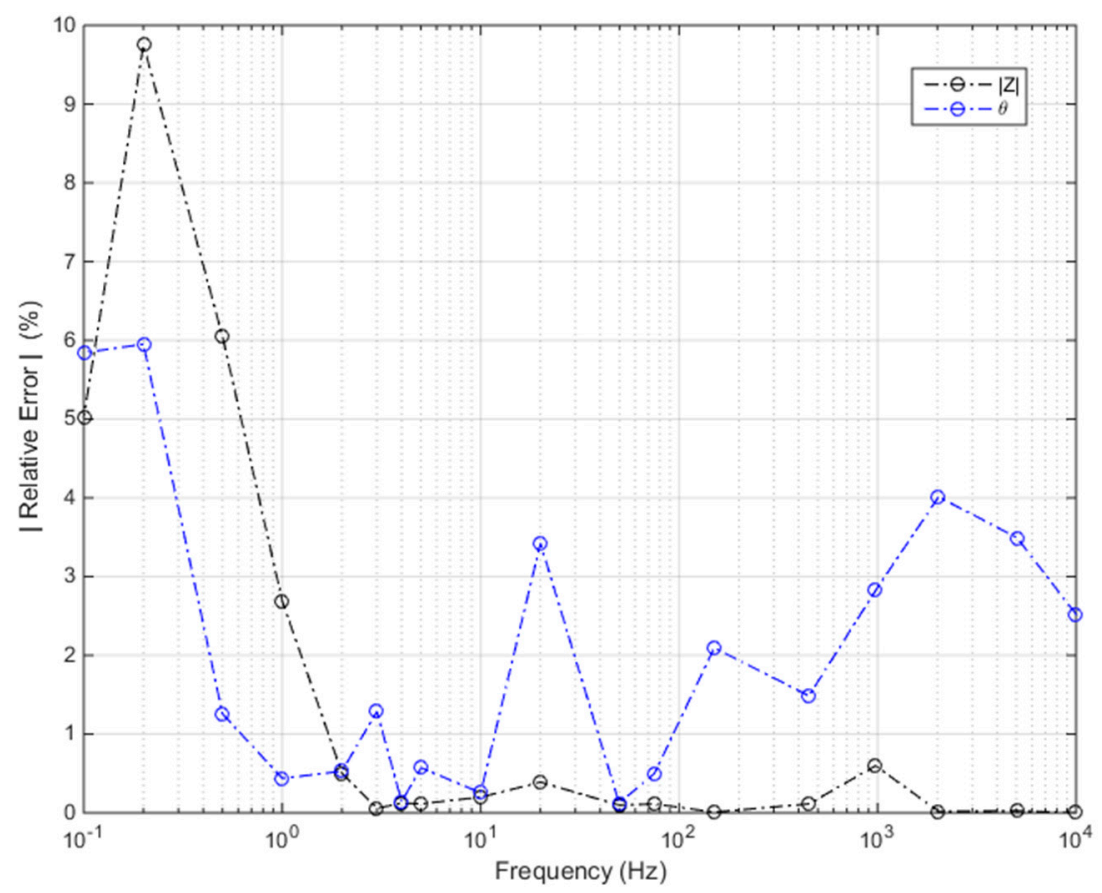

Figure 21. Relative error for estimation of impedance magnitude (black line) and phase (blue line).

\section{Discussion}

This paper has presented a compact multi-channel FRA-IS instrument that fully relies on a low cost Propeller multicore microcontroller, accomplishing a complete actuation-detection solution needing minimum additional external components. The excitation signal for impedance characterization is generated by a PDM-based generator running on a single core. This module generates up to two pairs of quadrature signals at a single frequency, so that the number of cells to be characterized at the same time can be highly extended by using adaptation modules (voltage followers in Figure 14) connected to the different signal generation ports. In this way, the (bio)impedance characterization processes can be highly parallelized, as it is demanded by current array-based applications. Signal recovery for impedance characterization is performed using the rest of available cores in the microcontroller, being possible to simultaneously acquire up to 7 impedance measurements, one per core. This number can be proportionally widened by extending the number of microcontrollers where the $\Sigma \Delta$ algorithm is implemented in the reading process, provided they receive the excitation and sensor output signals.

On the other hand, the proposed architecture allows impedance characterization using different excitation frequencies for several Randles cells in parallel, just assigning a different generation core per frequency. In fact, a more general solution could consist on assigning the cores of a Propeller microcontroller to generate the different frequencies (implementing the corresponding PDM and LPF per core), while using additional Propeller microcontrollers dedicated to the acquisition and impedance measurement tasks, implementing the $\Sigma \Delta$-ADC in each of the processor cores.

Therefore, the proposed EIS system constitutes a fully operative flexible and modular solution, suitable for multi-channel acquisition while complying the features of portability, and with an enhanced trade-off between low cost and measurement performance compared to similar devices in the literature. Reviewing the state-of-art, a direct approach relies on the use of the component AD5933 or the newer ADuCM350, which shown satisfactory results in different applications $[4,11]$, but performing one measurement process at a time, and at the cost of the high computing power required to implement the Discrete Fourier Transform (DFT) compared to the FRA technique. Similarly, comparable alternative low-cost microcontroller-based EIS architectures $[30,31]$ need more complex external hardware, providing typically worst resolution while covering a similar frequency range and for a single channel impedance measurement. Finally, Table 1 compares the implemented analyzer 
performances with those of previous multichannel implementations operating at a similar frequency range. It can be seen that our proposal achieves better resolution over a wider frequency range.

Table 1. Comparative analysis. EIS: Electrochemical impedance spectroscopy.

\begin{tabular}{cccc}
\hline Characteristic & [28] & [23] & This Work \\
\hline Technology & $0.5 \mathrm{~mm}$ & $0.13 \mathrm{~mm}$ & COTS \\
Supply voltage & $3.0 \mathrm{~V}$ & $1.2 \mathrm{~V}$ & $3.3 \mathrm{~V}$ \\
Signal Bandwidth & $10 \mathrm{mHz}$ to $100 \mathrm{~Hz}$ & $100 \mathrm{mHz}$ to $10 \mathrm{kHz}$ & $1.1 \mu \mathrm{Hz}$ to $10 \mathrm{kHz}$ \\
Channels & 100 & 16 & $7 /$ microcontroller \\
Waveform Generation & External & R-2R DAC & 2nd Order $\Delta$-DAC by PDM \\
Generator Resolution & $\mathrm{N} / \mathrm{A}$ & 8 -bit tuning & 14-bit coarse tuning + \\
Readout Structure & Lock-in IDC & DS-MADC & 1st order $\Sigma \Delta \mathrm{ADC}$ \\
Conversion rate & $10 \mathrm{kHz}$ & $10 \mathrm{kHz}$ & $20 \mathrm{kHz}$ \\
Effective number of bits & $8 \mathrm{bits}$ & $9.3 \mathrm{bits}$ & $12 \mathrm{bits}$ \\
(ENOB) & $\mathrm{N} / \mathrm{A}$ & $-44 \mathrm{~dB}$ & $-48.5 \mathrm{~dB}$ \\
THD worst case & $\mathrm{N} / \mathrm{A}$ & $8.4 \%$ & $10 \%$ \\
EIS max. relative error & & &
\end{tabular}

Therefore, the proposed approach succeeds in reducing instrument dimensions to allow automatic and in-situ multichannel impedance measurements, while improving the measurement performance using low-cost commercial components off the shelf (COTS).

Author Contributions: Conceptualization, A.S.-G., N.M. and B.C.; Methodology, N.M. and B.C.; hardware and software design and implementation, A.S.-G.; formal analysis, A.S.-G. and P.A.M.; experimental analysis, A.S.-G., N.M. and B.C.; writing-review and editing, N.M., B.C. and A.S.-G.; supervision, N.M. and B.C.

Funding: This research was funded by MINECO-FEDER, UE, grant number TEC2015-65750-R.

Acknowledgments: Authors would like to acknowledge the use of Servicio General de Apoyo a la Investigación-SAI, University of Zaragoza.

Conflicts of Interest: The authors declare no conflict of interest. The funders had no role in the design of the study; in the collection, analyses, or interpretation of data; in the writing of the manuscript, or in the decision to publish the results.

\section{References}

1. Randles, J.E.B. Kinetics of rapid electrode reactions. Discuss. Faraday Soc. 1947, 1, 1-11. [CrossRef]

2. Yang, L.; Bashir, R. Electrical/electrochemical impedance for rapid detection of foodborne pathogenic bacteria. Biotechnol. Adv. 2008, 26, 135-150. [CrossRef] [PubMed]

3. Liu, J.T.; Settu, K.; Tsai, J.Z.; Chen, C.J. Impedance sensor for rapid enumeration of E. coli in milk samples. Electrochim. Acta 2015, 182, 89-95. [CrossRef]

4. Durante, G.; Becari, W.; Lima, F.; Peres, H. Electrical Impedance Sensor for Real-Time Detection of Bovine Milk Adulteration. IEEE Sens. J. 2016, 16, 861-865. [CrossRef]

5. Sitkov, N.; Zimina, T.; Soloviev, A. Development of Impedimetric Sensor for E. coli M-17 Antibiotic Susceptibility Testing. In Proceedings of the IEEE Conference of Russian Young Researchers in Electrical and Electronic Engineering (EIConRus), Moscow, Russia, 29 January-1 February 2018.

6. Conesa, C.; Ibáñez Civera, J.; Seguí, L.; Fito, P.; Laguarda-Miró, N. An Electrochemical Impedance Spectroscopy System for Monitoring Pineapple Waste Saccharification. Sensors 2016, 16, 188. [CrossRef] [PubMed]

7. Mansoorifar, A.; Kokñu, A.; Ma, S.; Raj, G.; Beskok, A. Electrical Impedance Measurements of Biological Cells in Response to External Stimuli. Anal. Chem. 2018, 90, 4320-4327. [CrossRef]

8. Barsoukov, E.; Macdonald, J.R. Impedance Spectroscopy, Theory, Experiment and Applications, 3rd ed.; Wiley: Hoboken, NJ, USA, 2018; ISBN 9781119074083. 
9. Pérez-Navarrete, J.B. Establishment of Electrical Equivalent Circuits form Electrochemical Impedance Spectroscopy Study of Corrosion Inhibition of Steel by Imidazolium Derived Ionic Liquids in Sulphuric Acid Solution. In Proceedings of the 7th International Conference on Electrical Engineering, Computing Science and Automatic Control (CCE), Tuxtla Gutiérrez, México, 8-10 September 2010.

10. Alchaddoud, A.; Ibrahem, G.; Canale, L.; Zissis, G. Impedance spectroscopy and evolution of the equivalent circuit model for large area organic light emitting diodes aged under stress. In Proceedings of the 18th International Conference on Environmental and Electrical Engineering, Palermo, Italy, 12-15 June 2018.

11. Kaur, N.; Bhalla, S.; Shanker, R.; Panigrahi, R. Experimental Evaluation of Miniature Impedance Chip for Structural Health Monitoring of Prototype Steel/RC Structures. Exp. Tech. 2016, 40, 981-992. [CrossRef]

12. Deng, Z.; Zhang, Z.; Lai, Y.; Liu, J.; Li, J.; Liu, Y. Electrochemical Impedance Spectroscopy Study of a Lithium/Sulfur Battery: Modeling and Analysis of Capacity Fading. J. Electrochem. Soc. 2013, 160, A553-A558. [CrossRef]

13. Oprea, M.I.; Spataru, S.V.; Sera, D.; Poulsen, P.B.; Thorsteinsson, S.; Basu, R.; Andersen, A.R.; Frederiksen, K. Detection of Potential Induced Degradation in c-Si PV Panels Using Electrical Impedance Spectroscopy. In Proceedings of the 43rd IEEE Photovoltaic Specialists Conference (PVSC), Portland, OR, USA, 5-10 June 2016.

14. Li, H.; Liu, X.; Li, L.; Mu, X.; Genov, R.; Mason, A.J. CMOS Electrochemical Instrumentation for Biosensor Microsystems: A Review. Sensors 2017, 17, 74. [CrossRef] [PubMed]

15. Analog Devices. AD5933 Datasheet; Analog Devices: Norwood, MA, USA, 2017.

16. Rairigh, D.; Mason, A.; Chao, Y. Analysis of On-Chip Impedance Spectroscopy Methodologies for Sensor Arrays. Sens. Lett. 2006, 4, 398-402. [CrossRef]

17. Temiz, Y.; Lovchik, R.D.; Kaigala, G.V.; Delamarche, E. Lab-on-a-chip devices: How to close and plug the lab? Microelectron. Eng. 2015, 132, 156-175. [CrossRef]

18. Kusche, R.; Klimach, P.; Ryschka, M. A Multichannel Real-Time Bioimpedance Measurement Device for Pulse Wave Analysis. IEEE TBioCAS 2018, 12, 614-622. [CrossRef] [PubMed]

19. Valente, V.; Demosthenous, A. Wideband Fully-Programmable Dual-Mode CMOS Analogue Front-End for Electrical Impedance Spectroscopy. Sensors 2016, 16, 1159. [CrossRef] [PubMed]

20. Yúfera, A.; Rueda, A. A CMOS Bio-Impedance Measurement System. In Proceedings of the 12th International Symposium on Design and Diagnostics of Electronic Circuits \& Systems, Liberec, Czech Republic, 15-17 April 2009.

21. Onet, R.; Rusu, A.; Rodriguez, S. High-Purity and Wide-Range Signal Generator for Bioimpedance Spectroscopy. IEEE Trans. Circuits-II 2017, 1-5. [CrossRef]

22. Chen, T.; Wu, W.; Wei, C.; Darling, R.; Liu, B. Novel 10-bit Impedance-to-Digital Converter for Electrochemical Impedance Spectroscopy Measurements. IEEE TBioCAS 2017, 11, 370-379. [CrossRef] [PubMed]

23. Mazhab-Jafari, H.; Soleymani, L.; Genov, R. 16-Channel Impedance Spectroscopy DNA Analyzer With Dual-Slope Multiplying ADCs. IEEE TBioCAS 2012, 6, 468-478. [CrossRef]

24. Parallax Semiconductor. Propeller P8X32A Datasheet, Rev. 1.4. 6/14/2011; Parallax Semiconductor: Rocklin, CA, USA, 2011.

25. Parallax Semiconductor. Propeller P8X32A Counters Application Note AN001; Parallax Semiconductor: Rocklin, CA, USA, 2011.

26. Park, S. Principles of Sigma-Delta Modulation for Analog-to-Digital Converters; Motorola: Chicago, IL, USA, 2008.

27. Parallax Semiconductor. Sigma-Delta Analog to Digital Conversion Application Note AN008; Parallax Semiconductor: Rocklin, CA, USA, 2011.

28. Yang, C.; Jadhav, S.R.; Worden, R.M.; Mason, A.J. Compact Low-Power Impedance-to-Digital Converter for Sensor Array Microsystems. IEEE JSSC 2009, 44, 2844-2855. [CrossRef]

29. Burkhard, R.; Braach-Maksvytis, V.; Cornell, B.A.; King, L.G.; Osman, P.D.; Pace, R.J.; Wieczorek, L. Tethered Lipid Bilayer Membranes: Formation and Ionic Reservoir Characterization. Langmuir 1998, 14, 648-659. 
30. Corbellini, S.; Vallan, A. Arduino-based portable system for bioelectrical impedance measurement. In Proceedings of the IEEE International Symposium on Medical Measurements and Applications (IEEE MeMeA), Lisbon, Portugal, 11-12 June 2014.

31. Grassini, S.; Corbellini, S.; Angelini, E.; Ferraris, E.; Parvis, M. Low-cost impedance spectroscopy system based on logarithmic amplifier. IEEE Trans. Instrum. Meas. 2015, 64, 1110-1117. [CrossRef] 